\title{
Observables and Dynamics Quantum to Classical from a Relativity Symmetry and Noncommutative-Geometric Perspective
}

\author{
Chuan Sheng Chew, Otto C. W. Kong*, Jason Payne \\ Department of Physics and Center for High Energy and High Field Physics, National Central University, Taiwan \\ Email: *otto@phy.ncu.edu.tw
}

How to cite this paper: Chew, C.S., Kong, O.C.W. and Payne, J. (2019) Observables and Dynamics Quantum to Classical from a Relativity Symmetry and NoncommutativeGeometric Perspective. Journal of High Energy Physics, Gravitation and Cosmology, 5, 553-586.

https://doi.org/10.4236/jhepgc.2019.53031

Received: March 21, 2019

Accepted: May 6, 2019

Published: May 9, 2019

Copyright $\odot 2019$ by author(s) and Scientific Research Publishing Inc. This work is licensed under the Creative Commons Attribution International License (CC BY 4.0).

http://creativecommons.org/licenses/by/4.0/

\begin{abstract}
With approaching quantum/noncommutative models for the deep microscopic spacetime in mind, and inspired by our recent picture of the (projective) Hilbert space as the model of physical space behind basic quantum mechanics, we reformulate here the WWGM formalism starting from the canonical coherent states and taking wavefunctions as expansion coefficients in terms of this basis. This provides us with a transparent and coherent story of simple quantum dynamics where both the wavefunctions for the pure states and operators acting on them arise from the single space/algebra, which exactly includes the WWGM observable algebra. Altogether, putting the emphasis on building our theory out of the underlying relativity symmetry-the centrally extended Galilean symmetry in the case at hand-allows one to naturally derive both a kinematical and a dynamical description of a quantum particle, which moreover recovers the corresponding classical picture (understood in terms of the Koopman-von Neumann formalism) in the appropriate (relativity symmetry contraction) limit. Our formulation here is the most natural framework directly connecting all of the relevant mathematical notions and we hope it may help a general physicist better visualize and appreciate the noncommutative-geometric perspective behind quantum physics. It also helps to inspire and illustrate our perspective on looking at quantum mechanics and quantum physics in general in direct connection to the notion of quantum (deformed) relativity symmetries and the corresponding quantum/noncommutative models of spacetime as various levels of approximations all the way down to the Newtonian.
\end{abstract}

\section{Keywords}

Dynamics Quantum, Relativity Symmetry, WWGM 


\section{Introduction to the Quantum Relativity Perspective}

Some years before the turn of the century, the idea that physical spacetime should be modeled, at least at the deep microscopic scale, by some form of noncommutative geometry [1] started to get more and more appreciation from physicists. A major part of it has to do with considerations about the compatibility of the basic notion is quantum mechanics and a theory of geometrodynamics like Einstein general relativity [2]. Noncommutative geometry being obtainable from string theory [3] also helps to promote the idea. The development of noncommutative geometry in mathematics has been driven however in quite a part by the study of operator algebras as motivated by the observable algebra of quantum mechanics. Quantum mechanics is about noncommutative geometry though the observable algebra is usually taken as related only to the quantum phase space. The Newtonian space is still assumed to be the right model for the physical space. Yet, the position observable which should be like coordinates in the space is given by elements of the noncommutative observable algebra. In a theory of particle dynamics, the only truly physical notion about the space is really the totally of all possible positions for a free particle. The Newtonian space is the configuration space for a Newtonian free particle, and the phase space is like a sum of two copies, as the momentum space is isomorphic to the configuration space. In fact, one can start from the relativity symmetry group, the Galilean $G(3)$ group in the case, and obtain the spaces from a representation of the latter, deriving the dynamics as symmetry flows generated by the Hamiltonian as the energy observable. For quantum mechanics, there is no notion of configuration space and the phase space can only be obtained from the so-called projective representation of $G(3)$, which really means a unitary representation of a bigger group, the $U(1)$ central extension $\tilde{G}(3)$. Our key perspective is that the latter should be taken as the relativity symmetry behind quantum mechanics and the phase space taken as the quantum model for the physical space. In Ref. [4], we have given a brief report on the key result that the perspective not only put the related notions of quantum mechanics on the same footing as those for Newtonian mechanics, one can also retrieve the Newtonian ones, including the Newtonian space, as classical limits of the quantum notions. The current paper presents the full picture of the dynamics. The nonexistence of any useful notion of the quantum configuration space, or momentum space, here is simply a direct parallel of the nonexistence of independent notion of space and time in the Einstein theory. The quantum phase space as the quantum physical space only splits into the configuration and momentum space at the Newtonian limit.

Our background theoretical/mathematical setting is given by that of deformed special relativity [5]-[15], within a Lie algebra/group framework [4] [16] [17] [18]. The key theme of deformed special relativity is to look at alternative theories of special relativity, including possible candidates for deep microscopic physics with interesting features including noncommutativity, to which the Einstein theory is an approximation. Note that the notion of spacetime non- 
commutativity at the zero gravity limit has no conflict at all with necessity of it in the presence of gravity. Our formulation shows a noncommutative model for the physical space is already there behind basic quantum mechanics. It is essentially a deformation of the Newtonian, with deformation here taken on the level of the relativity symmetry. The latter exerts its effects to the spacetime model [4] and every aspects of the dynamical theory. We present, however, the inversedeformation as symmetry contractions [16]. The fully deformed/stabilized level has full noncommutativity [17], all without considering gravity. The theory with gravity would be the "general quantum relativity", in which we expect the noncommutativity to be the quantum gravitational dynamics [18] [19]. Results of ours then suggests any such models should probably include the case of quantum mechanics at some limit before getting to the Newtonian.

A fundamental idea behind our approach lies in obtaining a fully grounded picture with a definite starting point of noncommutativity in physics, namely simple quantum mechanics and its commutative limit. The current paper actually presents only results relating to the latter part. The mathematics of noncommutative geometry starts from a (noncommutative) algebra, and the algebra of observables for quantum mechanics with the Heisenberg structure is, of course, noncommutative. This noncommutativity is, however, only between the position and momentum observables; thus, one may think that the noncommutative-geometrical picture lurking behind quantum mechanics can only be interpreted as some sort of phase space, rather than a space(time). For a theory of particle dynamics, physical space is observable only as the space of all possible positions for a free particle, or (more generally) the center of mass for a closed system of particles, i.e. the configuration space. There is, however, no notion of configuration space in quantum mechanics. In the classical case, we have the configuration space being given by "half" of the phase space; therefore, it makes good sense to then look carefully at the phase space. Our analysis from the relativity symmetry perspective [4] answers this question well: we have illustrated that the Hilbert space-as the quantum phase space-can be seen as reducing to either the Newtonian phase space or configuration space in the classical limit, formulated as the symmetry contraction limit. Such a contraction is the "inverse" of a relativity symmetry deformation. To put it another way, the phase space is an irreducible representation under the quantum relativity symmetry, while the classical phase space is reducible to the configuration space and momentum space parts. Einsteinian special relativity says Newtonian space and time are only parts of Minkowski spacetime, and can be handled separately only under the Newtonian approximation. Our result gives a similar picture regarding the position and momentum parts of the phase space. Consequently, the phase space is the right model for physical space. Some other authors working on noncommutative spacetime have indeed brought in a symplectic or Poisson structure [20] for noncommutative spacetime, through not connecting the latter with the phase spaces of classical and quantum mechanics. Our perspective here suggests thinking more about deformation of spacetime and its dynamics through deformation 
of what used to be called phase space instead of what is essentially only the configuration space part. It is interesting to note that the notion of the phase space as the inseparable model of the physical spacetime in quantum settings actually has a parallel in the recent considerations of Born reciprocity and doubled geometry from string theory [21]. It remains to see though if there may be the necessity to go beyond the symplectic and Riemannian geometric setting from our framework.

The projective Hilbert space, as the space of pure states, is a dual geometric structure to the observable algebra [22]. It is still a commutative manifold, but an infinite-dimensional one. We consider it an alternative, real-number geometric description of the noncommutative geometry. The quantum space behind quantum mechanics may be described by the six $\hat{X}_{i}$ and $\hat{P}_{i}$ noncommutative coordinate observables or the infinite set of real number coordinates of the projective Hilbert space. Both sets essentially reduce to the same classical limit of the Newtonian phase space coordinates $x_{i}$ and $p_{i}$.

The Weyl-Wigner-Groenewold-Moyal (WWGM) formalism [23] [24] [25] [26] [27], or deformation quantization, is a key approach for passing from the commutative to the noncommutative. Such a deformation quantization of coordinate observables has been a theme in the construction of noncommutative spacetime. The present paper, however, rewrites the WWGM formalism from the relativity symmetry perspective, i.e. from the cyclic irreducible representation of the group $C$-algebra corresponding the the unitary representation of the group on the Hilbert space. The classical limit is retrieved as the symmetry contraction limit pushing all the way to the algebra of observables and their dynamical evolution. Deformation quantization is therefore established as the deformation of the representation of the group $C$-algebra, arising as a consequence of the relativity symmetry deformation. We see this work as providing the crucial first link from the bottom-up to any quantum/noncommutaive models of spacetime. It also suggests looking at quantum physics from a noncommutativegeometric point of view. Most, if not all, of the mathematics presented in the paper is essentially there scattered in the physics and mathematics literature. Our work is to pull all that together under an explicit consistent framework to illustrate our $\tilde{G}(3)$ relativity perspectives for the particle dynamics as one on the quantum model of the physical space, and the full passage to $G(3)[\otimes U(1)]$ Newtonian limit.

A word on some of the mathematics not otherwise addressed here may be in order. Deformation quantization in the some references to noncommutative geometry is written in the language of the twisting of a Hopf algebra structure. Nothing like the latter has been used in the case of basic quantum mechanics, which is the case considered in the analysis presented in this article. That said, the group $C$-algebra for the Heisenberg-Weyl group, or its irreducible representations, has a natural (twisted) convolution as a noncommutative product to which the Moyal star-product is essentially a Fourier transform. The group $C$-algebra can also be promoted to a Hopf algebra by a simple commutative co- 
product, as such it is dual to the Hopf algebra with the commutative pointwise function product. It also possesses a natural noncommutative coproduct [28]. In the commutative contraction limit of the group, all of these products and coproducts are, of course, commutative.

\section{WWGM Formalism as Representation of Group C*-Algebra Corresponding to Coherent State Representation}

The Weyl-Wigner-Groenewold-Moyal (WWGM) formalism [20] [24] [25] [26] [27] describes quantum mechanics on the space of tempered distributions on the set of variables, usually taken as coordinates of the classical phase space. Physical observables, as hermitian operators on the Hilbert space of pure states, are described by real functions. A quantum state, pure or mixed, corresponds exactly to a function for the density operator. A noncommutative product-the Moyal star product-gives the space of generalized observables the structure of an algebra. The Moyal algebra for physical observables is isomorphic to the corresponding algebra of operators and reduces to the Poisson algebra of classical observables in the $\hbar \rightarrow 0$ limit. While the formalism provides a complete description of quantum mechanics without the need for the usual wavefunctions, it is usually-and especially historically-introduced through the Weyl-Wigner transform starting from Schrödinger wavefunction over position variables. Such wavefunctions is only background used for deriving the results, but is not an integral part of the formulation. The (canonical) coherent state [29] [30] [31] [32] representation provides an alternative giving the Schrödinger wavefunction as a function on essentially "classical" phase space variables much similar to the operators in WWGM itself. The symplectic manifold of the set of classical states can be seen as the submanifold of the basis coherent states in the infinite-dimensional Kähler manifold of the quantum projective Hilbert space [33]. Moreover, there is the Koopman-von Neumann formulation of classical mechanics in the language of Hilbert spaces [34] [35] [36]. From all of these, we expect looking at the WWGM formalism through the Weyl-Wigner transform starting instead from the Hilbert space of wavefunctions over the coherent state basis will provide a particularly interesting picture of quantum mechanics which would also be suitable for the analysis of the classical limit. One would then have operators and states both plausibly described by elements in the same space of functions or distributions. Consequently, both the WWGM and the Hilbert space formalisms may be unified as one. We are not aware of any explicit treatment along these lines; hence we present one in this article (important work for the coherent states has, however, been given in Ref. [37]). A key feature, for example, is that a function $\alpha$ acts by the star product $\alpha \star$ as an operator on a wavefunction $\phi$; the Moyal star product $\alpha \star \beta$ between functions is essentially the operator product $\alpha \star \beta \star$. In other words, the wavefunctions we start with end up as objects inside the Moyal algebra(s) of "observables"; the Hilbert space is nothing other than the one obtained through an algebraic GNS construction 
[38] [39] from the Moyal algebra itself.

In a recent paper [4], we have introduced the idea of looking at the central extension of the Galilean group as the true relativity symmetry group for quantum mechanics and the Newtonian case as the contraction limit of the symmetry [16] [40] [41] trivializing the central extension. The Hilbert space serves as the phase space, and in a way also the configuration space, of a free quantum particle. Taken as representation spaces of the relativity symmetry, the limit of the two picture under the contraction can be traced to give what are essentially the phase and configuration space of the classical Newtonian picture. The latter is more directly given, naively, in the language of Hilbert spaces; hence the Koopman-von Neumann formulation-a part of the story to be described explicitly here. The coherent states serve as the basis for the construction of the quantum Hilbert space representation and are the only ones surviving as (pure) states in the classical limit. The current study is partly motivated, therefore, by the need for a corresponding full dynamical picture of this story. Indeed, the Heisenberg picture provides a more transparent illustration of the dynamics. The WWGM formalism is, of course, supposed to focus on the observables more than the pure states as vectors in a Hilbert space. The coherent state formulation, however, makes the pure states directly accessible within the same algebraic framework. This framework gives a solid setting for the analysis of time evolution both within and outside of the relativity symmetry perspective. All symmetries can be described on a similar footing, as unitary transformations on the Hilbert space and automorphisms on the algebra of observables.

We especially want to emphasize here our perspective that all of the mathematical structures behind the physical theory naturally manifest themselves from the (relativity) symmetry group and its associated structures. The Hilbert space of pure states is an irreducible unitary representation of the group and the observable algebra is an irreducible representation of the group ( $C$-)algebra. The former as an irreducible representation of the latter, within our framework, sits naturally inside the latter; the natural (noncommutative algebraic) multiplicative actions of which is the operator action. For a somewhat parallel picture for describing mixed states, we bring in the notion of a Tomita representation [42] [43], which sees a density matrix (for a mixed state) as a vector in a Hilbert space (of operators). This is particularly useful for describing symmetry in the Koopman-von Neumann formulation in the symmetry contraction limit. Symmetries are represented as unitary transformations on the Hilbert spaces and inner automorphisms of the observable algebra. The dynamical picture naturally follows. So, the analysis here establishes explicitly that the $\tilde{G}(3)$ plays the complete role of a relativity symmetry to quantum mechanics and give the classical approximation of the Newtonian case in all corresponding aspects.

In light of the above, the basic perspective of our framework is to start with the coherent state representation, essentially seen as a representation of the extended Galilean symmetry, which is equivalent to the one formulated simply 
with the Heisenberg-Weyl group [4], and the classical theory is to be retrieved through the contraction limit. We write quantum expressions with the $\hbar=2$ units here, following Ref. [25]. This choice gives the Heisenberg commutation algebra the "unusual" form of

$$
\left[X_{i}, P_{j}\right]=2 i \delta_{i j} I,
$$

while setting the minimal uncertainty product to unity. It is in many ways the more natural choice of units for quantum theory. The next section sets the stage for the Hilbert space description. The explicit WWGM formalism is presented in Section 4 . We emphasize the group theoretical aspects, and give more details only for results and features specific to the coherent state framework. Section 5 presents the symmetry contraction to the classical theory. The Tomita representation is introduced at the end of the section. In Section 6, we summarize the description of quantum symmetries and work out explicit results for elements of the relativity symmetry, including results on the Hilbert space of the Tomita representation. Dynamics is described by a time (translation symmetry) transformation. The section following this traces the symmetry descriptions to the contraction limit, and Section 8 focuses on the classical dynamical picture a la Koopman-von Neumann (and otherwise). The last section presents some concluding remarks.

\section{The Coherent State Representation}

We start with the familiar (canonical) coherent state representation

$$
\left|p^{i}, x^{i}\right\rangle=U\left(p^{i}, x^{i}\right)|0\rangle \equiv \mathrm{e}^{-i \theta} U\left(p^{i}, x^{i}, \theta\right)|0\rangle
$$

where

$$
U\left(p^{i}, x^{i}, \theta\right) \equiv \mathrm{e}^{i x_{i} p^{i} \hat{I}} \mathrm{e}^{i \theta \hat{I}} \mathrm{e}^{-i x^{i} \hat{P}_{i}} \mathrm{e}^{i p^{i} \hat{X}_{i}}=\mathrm{e}^{i\left(p^{i} \hat{X}_{i}-x^{i} \hat{P}_{i}+\theta \hat{I}\right)},
$$

$|0\rangle \equiv|0,0\rangle$ is a fiducial normalized vector, $\hat{X}_{i}$ and $\hat{P}_{i}$ are representations of the generators $X_{i}$ and $P_{i}$ as self-adjoint operators on the abstract Hilbert space $\mathcal{H}$ spanned by the $\left|p^{i}, x^{i}\right\rangle$ vectors, and $\hat{I}$ is the identity operator representing the central generator $I .\left(p^{i}, x^{i}, \theta\right)$ corresponds to a generic element of the Heisenberg-Weyl group as

$$
W\left(p^{i}, x^{i}, \theta\right)=\exp \left[i\left(p^{i} X_{i}-x^{i} P_{i}+\theta I\right)\right],
$$

with

$$
W\left(p^{\prime i}, x^{\prime i}, \theta^{\prime}\right) W\left(p^{i}, x^{i}, \theta\right)=W\left(p^{i i}+p^{i}, x^{\prime i}+x^{i}, \theta^{\prime}+\theta-\left(x_{i}^{\prime} p^{i}-p_{i}^{\prime} x^{i}\right)\right) .
$$

Here, $\left(x_{i}^{\prime} p^{i}-p_{i}^{\prime} x^{i}\right)$ is the classical mechanical symplectic form. Note that we have $p^{i}$ and $x^{i}$ here corresponding to half the expectation values of $\hat{P}_{i}$ and $\hat{X}_{i}$ ( $\hbar=2$ units). In the interest of simplifying the notation, we drop the index $i$ in most of the subsequent expressions, only putting it back explicitly when some emphasis on the three-vector nature of a given quantity is warranted.

We introduce wave functions on the coherent state manifold 
$\phi(p, x) \equiv\langle p, x \mid \phi\rangle$ with

$$
\begin{aligned}
& \langle p, x|\hat{X}| \phi\rangle=\hat{X}^{L} \phi(p, x), \\
& \langle p, x|\hat{P}| \phi\rangle=\hat{P}^{L} \phi(p, x),
\end{aligned}
$$

where

$$
\begin{aligned}
& \hat{X}^{L}=x+i \partial_{p}, \\
& \hat{P}^{L}=p-i \partial_{x},
\end{aligned}
$$

and

$$
U^{L}(p, x) \phi\left(p^{\prime}, x^{\prime}\right) \equiv\left\langle p^{\prime}, x^{\prime}|U(p, x)| \phi\right\rangle=\phi\left(p^{\prime}-p, x^{\prime}-x\right) \mathrm{e}^{i\left(p x^{\prime}-x p^{\prime}\right)} .
$$

Furthermore, we have a realization of the quantum Hilbert space as a closed (polarization) subspace of $L^{2}(\Pi)$, the space of the wavefunctions $\phi(p, x)$ on which $U^{L}$ acts as a (projective) representation of the Heisenberg-Weyl group. We can see again that $\hat{P}^{L}$ and $\hat{X}^{L}$ generate translations in $x$ and $p$, respectively. The wavefunction $\phi_{a}(p, x)$ for the coherent state $\left|p_{a}, x_{a}\right\rangle$ is given by

$$
\phi_{a}(p, x) \equiv\left\langle p, x \mid p_{a}, x_{a}\right\rangle=\mathrm{e}^{i\left(p_{a} x-x_{a} p\right)} \mathrm{e}^{-\frac{1}{2}\left[\left(p-p_{a}\right)^{2}+\left(x-x_{a}\right)^{2}\right]} ;
$$

in particular, the $|0,0\rangle$ state wavefunction is denoted by $\phi_{0}(p, x)$ and $\phi_{0}(p, x)=\mathrm{e}^{-\frac{1}{2}\left(p^{i} p_{i}+x^{i} x_{i}\right)}$, which is a symmetric Gaussian of unit width. The expression $\left\langle p, x \mid p_{a}, x_{a}\right\rangle$ may also be taken as giving the overlap of two different coherent states. $\phi_{a}(p, x)$ is a test function belonging to the Schwartz space of smooth rapidly decreasing functions $S(\Pi)$. In what follows, we will denote the Hilbert space of wavefunctions by $\mathcal{K}$. The representations, with or without the superscript $L$ (i.e. on $\mathcal{K}$ or on $\mathcal{H}$ ), are of course unitarily equivalent. The natural inner product on $\mathcal{K}$ is $\frac{1}{\pi^{n}} \int \mathrm{d} p \mathrm{~d} x \bar{\phi}(p, x) \phi^{\prime}(p, x)=\left\langle\phi|\hat{I}| \phi^{\prime}\right\rangle$ with $\hat{I}=\frac{1}{\pi^{n}} \int \mathrm{d} p \mathrm{~d} x|p, x\rangle\langle p, x|$, which keeps $\phi_{a}(p, x)$ as a normalized wavefunction. Note that we use $n$ for the dimension of the classical physical space, though we only consider $n=3$ here.

For a full discussion of the algebra of smooth observables, we will go beyond the Hilbert space of pure states for the limited class of bounded observables. Pure states for smooth observables are unit rays in $\mathcal{S} \equiv S(\Pi) \cap \mathcal{K} \quad$ [26], though at times we may not pay full attention to the difference below.

\section{The Observable Algebra from the WWGM Formalism}

In this article, we emphasize the key role of the associated structures of the symmetry group behind the physical theory. We have seen that the Heisenberg-Weyl group manifold, or the isomorphic coset space of the extended Galilean group, provides a direct description of the coherent state basis [4] for the Hilbert space. Here, we see how the group ring provides a description of the set 
of operators and the observable algebra. The set of operators

$$
\Omega^{\prime}[\alpha(p, x, \theta)]=\frac{1}{(2 \pi)^{n+1}} \int \mathrm{d} p \mathrm{~d} x \mathrm{~d} \theta \alpha(p, x, \theta) U(p, x, \theta),
$$

where $\alpha(p, x, \theta)$ is a distribution on the group manifold, is a Heisenberg-Weyl ring [23]. Consider $\alpha(p, x) \equiv \alpha^{1}(p, x)$ in the expansion

$$
\alpha(p, x, \theta)=\int \mathrm{d} \lambda|\lambda|^{n} \alpha^{\lambda}(p, x) \mathrm{e}^{-i \lambda \theta} .
$$

It can easily be seen that the $\theta$-integration in $\Omega^{\prime}[\alpha(p, x, \theta)]$ can be performed to give a $2 \pi \delta(\lambda-1)$, which in turn yields $\Omega^{\prime}[\alpha(p, x, \theta)]=\Omega[\alpha(p, x)]$ with

$$
\Omega[\alpha(p, x)]=\frac{1}{(2 \pi)^{n}} \int \mathrm{d} p \mathrm{~d} x \alpha(p, x) U(p, x) .
$$

$\lambda$ can be interpreted as an eigenvalue of the central charge generator which is always unity under the representation. We have the latter as a continuous linear injection from $L^{1}(\Pi)$ into $\mathbb{B}(\mathcal{H})$. As such, it is a *algebra homomorphism with respect to the twisted convolution product $\circ$ and the involution $*$ defined by

$$
\alpha \circ \beta(p, x)=\frac{1}{(2 \pi)^{n}} \int \mathrm{d} p^{\prime} \mathrm{d} x^{\prime} \alpha\left(p^{\prime}, x^{\prime}\right) \beta\left(p-p^{\prime}, x-x^{\prime}\right) \mathrm{e}^{i\left(p^{\prime} x-x^{\prime} p\right)}
$$

and

$$
\alpha^{*}=\overline{\alpha(-p,-x)},
$$

respectively, where $\bar{\alpha}$ is the complex conjugate of $\alpha$. That is, $\Omega[\alpha \circ \beta]=\Omega[\alpha] \Omega[\beta]$ and $\Omega\left[\alpha^{*}\right]=\Omega[\alpha]^{\dagger}$. Self-adjoint elements of $\mathbb{B}(\mathcal{H})$ and their counterparts in $L^{1}(\Pi)$ represent the bounded observables. Here, $\Pi$ is the $(p, x)$-space, which can be considered as the coherent state manifold and is also the "classical phase space" $\mathbb{R}^{2 n}$ on which we have the wavefunctions $\phi(p, x)$. Note that the $\Omega$ map takes a delta function $\delta_{a}$ of mass 1 centered on $\left(p_{a}, x_{a}\right)$ to $U\left(p_{a}, x_{a}\right)=\mathrm{e}^{i\left(p_{a} \hat{X}-x_{a} \hat{P}\right)}$, and $\alpha \circ \delta_{o}=\delta_{o} \circ \alpha=\alpha$ for the delta function $\delta_{o}$ centered on $(0,0)$. The inverse mapping can be written as

$$
\alpha(p, x)=2^{n} \operatorname{Tr}\left[\Omega[\alpha] U^{\dagger}(p, x)\right],
$$

where the trace is to be evaluated over the set of coherent states as $\frac{1}{\pi^{n}} \int \mathrm{d} p^{\prime \prime} \mathrm{d} x^{\prime \prime}\left\langle p^{\prime \prime}, x^{\prime \prime}|\cdot| p^{\prime \prime}, x^{\prime \prime}\right\rangle$ and we have

$$
\operatorname{Tr}\left[U\left(p^{\prime}, x^{\prime}\right) U^{\dagger}(p, x)\right]=\pi^{n} \delta\left(p^{\prime}-p, x^{\prime}-x\right)\left\langle p, x \mid p^{\prime}, x^{\prime}\right\rangle .
$$

Similarly, we have

$$
\Omega^{L}\left[\alpha\left(p^{\prime}, x^{\prime}\right)\right]=\frac{1}{(2 \pi)^{n}} \int \mathrm{d} p^{\prime} \mathrm{d} x^{\prime} \alpha\left(p^{\prime}, x^{\prime}\right) U^{L}\left(p^{\prime}, x^{\prime}\right),
$$

where $U^{L}\left(p^{\prime}, x^{\prime}\right)=\mathrm{e}^{i\left(p^{\prime} \hat{X}^{L}-x^{\prime} \hat{p}^{L}\right)}=\mathrm{e}^{\left.i\left(p^{\prime} x-x^{\prime}\right)\right)} \mathrm{e}^{-\left(p^{\prime} \partial_{p}+x^{\prime} \partial_{x}\right)}$, with the set of $\Omega^{L}[\alpha]$ considered as operators on $L^{2}(\Pi)$ satisfying $\Omega^{L}[\alpha \circ \beta]=\Omega^{L}[\alpha] \Omega^{L}[\beta]$. Naturally ${ }^{1}$ ${ }^{1}$ Here $\operatorname{Tr}\left[\hat{\alpha}^{(L)}\right]=\frac{1}{\pi^{n}} \int \mathrm{d} p_{a} \mathrm{~d} x_{a} \bar{\phi}_{a} \hat{\alpha}^{(L)} \phi_{a}$. 


$$
\alpha(p, x)=2^{n} \operatorname{Tr}\left[\Omega^{L}[\alpha] U^{L \dagger}(p, x)\right] .
$$

In fact, the left-invariant vector fields, or differential operator realization of the generators, of the group manifold

$$
\begin{gathered}
X^{L}=i x \partial_{\theta}+i \partial_{p}, \\
P^{L}=i p \partial_{\theta}-i \partial_{x}, \\
I^{L}=i \partial_{\theta},
\end{gathered}
$$

have their action on a function $\alpha(p, x, \theta)$ in the form of Equation (11) given by the action on $\alpha^{\lambda}(p, x)$ defined by $\lambda x+i \partial_{p}, \lambda p-i \partial_{p}$, and 0 , respectively. Hence, $\lambda=1$ yields the differential operators $\hat{X}^{L}$ and $\hat{P}^{L}$ of Equation (7), as in $U^{L \dagger}(p, x)$, which are exactly the corresponding operators acting on $\alpha(p, x)\left[\equiv \alpha^{1}(p, x)\right]$.

The symplectic Fourier transform

$$
\alpha_{f}(p, x)=F[\alpha](p, x) \equiv \frac{1}{(2 \pi)^{n}} \int \mathrm{d} p^{\prime} \mathrm{d} x^{\prime} \alpha\left(p^{\prime}, x^{\prime}\right) \mathrm{e}^{i\left(p^{\prime} x-x^{\prime} p\right)} .
$$

is a continuous isomorphism of $S(\Pi)$, as a Fréchet space, onto itself extending to a unitary transformation on $L^{2}(\Pi)$ with $F^{2}=1$. The twisted product $\star$ satisfies

$$
F[\alpha] \star F[\beta]=F[\alpha \circ \beta]
$$

and $^{2}$

$$
F^{-1}[\alpha \star \beta]=F^{-1}[\alpha] \circ F^{-1}[\beta],
$$

with $1 \star \alpha=\alpha=\delta_{o} \circ \alpha$, in which case the two products commute. We also have

$$
\alpha \circ \beta=F[\alpha] \star \beta .
$$

This is the usual Moyal star product, which can be written as

$$
\alpha \star \beta(p, x)=\alpha(p, x) \mathrm{e}^{-i\left(\partial_{p} \vec{\partial}_{x}-\partial_{x} \vec{\partial}_{p}\right)} \beta(p, x),
$$

or in the integral form

$$
\begin{aligned}
& \alpha \star \beta(p, x) \\
& =\frac{1}{(2 \pi)^{2 n}} \int \mathrm{d} p^{\prime} \mathrm{d} x^{\prime} \mathrm{d} p^{\prime \prime} \mathrm{d} x^{\prime \prime} \alpha\left(p^{\prime}, x^{\prime}\right) \beta\left(p^{\prime \prime}, x^{\prime \prime}\right) \mathrm{e}^{-i\left(p x^{\prime}-x p^{\prime}\right)} \mathrm{e}^{i\left(p x^{\prime \prime}-x p^{\prime \prime}\right)} \mathrm{e}^{i\left(p^{\prime \prime} x^{\prime}-x^{\prime \prime} p^{\prime}\right)} .
\end{aligned}
$$

In particular, we have

$$
\begin{aligned}
& x \star \alpha=\left(x+i \partial_{p}\right) \alpha=\hat{X}^{L} \alpha, \\
& p \star \alpha=\left(p-i \partial_{x}\right) \alpha=\hat{P}^{L} \alpha .
\end{aligned}
$$

The Fourier transform $F$ is a continuous ${ }^{*}$-algebra isomorphism between $[S(\Pi), \circ, \dagger]$ and $\left[S(\Pi), \star_{-}^{-}\right]$, the latter involution being simple complex conjugation. Both the $\star$ and $\circ$ products can be extended to the space $S^{\prime}(\Pi)$ of ${ }^{2}$ We use $F^{1}$ instead of simply $F$ to keep track of difference which only manifests at the classical contraction limit discussed in the next section. 
tempered distributions. Notice that $F$ is more commonly written as a transform between functions of two different spaces, one being the parameter space for the Heisenberg-Weyl group modulo $\theta$, while the other is identified as the classical phase space, or rather the variable space of the Moyal star functional algebra. Our perspective of looking at quantum mechanics and its classical limit, focusing on the coherent state picture [4], may be considered a justification for identifying the two, as done in Refs. [24] [25] for example, at the quantum level. The "classical phase space" then is also the coherent state manifold with parameters characterizing, however, half the position and momentum expectation values ${ }^{3}$.

Consider

$$
\begin{aligned}
\Delta^{(L)}[\alpha] & \equiv \Omega^{(L)}\left[F^{-1}[\alpha]\right] \\
& =\frac{1}{(2 \pi)^{2 n}} \int \mathrm{d} p^{\prime} \mathrm{d} x^{\prime} \mathrm{d} p \mathrm{~d} x \alpha(p, x) \mathrm{e}^{i\left(p x^{\prime}-x p^{\prime}\right)} U^{(L)}\left(p^{\prime}, x^{\prime}\right) \\
& =\frac{1}{(2 \pi)^{n}} \int \mathrm{d} p \mathrm{~d} x \alpha(p, x) \Delta_{p, x}^{(L)},
\end{aligned}
$$

where we have ${ }^{4}$

$$
\Delta_{p, x}^{(L)}=\frac{1}{(2 \pi)^{n}} \int \mathrm{d} p^{\prime} \mathrm{d} x^{\prime} \mathrm{e}^{i\left(p x^{\prime}-x p^{\prime}\right)} U^{(L)}\left(p^{\prime}, x^{\prime}\right) .
$$

In these expressions, we are putting the two cases, with and without the superscript $L$, in a single set. This is the Weyl correspondence, i.e. we have $\hat{\alpha}^{L} \equiv \alpha\left(\hat{P}^{L}, \hat{X}^{L}\right)=\Delta^{L}[\alpha(p, x)]$ and $\hat{\alpha} \equiv \alpha(\hat{P}, \hat{X})=\Delta[\alpha(p, x)]$ with, however, $\hat{\alpha}^{L}$ here thought of as operators on wavefunctions on the manifold of coherent states. Then the bijective map $\Delta^{L}$ takes $S^{\prime}(\Pi)$ to $\mathcal{L}\left(S(\Pi), S^{\prime}(\Pi)\right)$, and we have

$$
\alpha(p, x)=2^{n} \operatorname{Tr}\left[\hat{\alpha}^{(L)} \Delta_{p, x}^{(L) \dagger}\right]
$$

Moreover, $\Delta^{(L)}[\alpha \star \beta(p, x)]=\Delta^{(L)}[\alpha(p, x)] \Delta^{(L)}[\beta(p, x)]$ gives a *-algebra isomorphism between the Moyal algebra $[\mathcal{M}, \star,-]$ and the corresponding algebra of smooth "observables" $\mathcal{L}^{+}(S(\Pi))$, with $\mathcal{M} \equiv\left\{\beta \in S^{\prime}(\Pi): \beta \star \alpha, \alpha \star \beta \in S(\Pi) \forall \alpha \in S(\Pi)\right\} ;$ and between $\left[\mathcal{M}^{\prime}, \star,-\right]$ and $\mathcal{L}^{+}\left(S(\Pi), L^{2}(\Pi)\right)$ as algebra of bounded "observables", with $\mathcal{M}^{\prime}:=\left\{\beta \in S^{\prime}(\Pi): \beta \star \alpha, \alpha \star \beta \in L^{2}(\Pi) \forall \alpha \in L^{2}(\Pi)\right\}$. Note that $\Delta[\bar{\alpha}(p, x)]=\Delta[\alpha(p, x)]^{\dagger}$; hence physical observables with $\hat{\alpha}=\hat{\alpha}^{\dagger}$, are given ${ }^{3}$ Note that though it looks like we have inconveniently made the group parameters and the coherent state expectation values differ by a factor of 2 by using $\hbar=2$ instead of $\hbar=1$ units, it is really results like Equation (26) that naturally prefer the convention. The parameter space for the wavefunctions $\phi$ can be exactly identified with that of the Moyal star functional algebra.

${ }^{4}$ We have $\Delta_{p, x}^{(L)}=U^{(L)}(p, x) \Delta_{0,0}^{(L)}$ with $\Delta_{0,0}^{(L)}$ being the phase space parity operator of GrossmannRoyer [44] [45]; i.e. $\Delta_{0,0}\left|p^{\prime}, x^{\prime}\right\rangle=\left|-p^{\prime},-x^{\prime}\right\rangle$ and $\Delta_{0,0}^{L} \phi\left(p^{\prime}, x^{\prime}\right)=\phi\left(-p^{\prime},-x^{\prime}\right)$. Note that $\Delta_{p, x}^{(L)}$ is actually selfadjoint, besides being unitary. 
by real elements of the Moyal algebras. We will, however, mostly not pay much attention to the difference between $\mathcal{M}$ and $\mathcal{M}^{\prime}$ below.

We define $(\star-)$ multiplicative operators acting on the distributions by

$$
M_{\star}[\alpha] \equiv \alpha \star .
$$

Then, we have the simple and elegant result

$$
M_{\star}[\alpha(p, x)]=\Delta^{L}[\alpha(p, x)]=\hat{\alpha}^{L}=\alpha\left(\hat{P}^{L}, \hat{X}^{L}\right)
$$

which can be interpreted as the Bopp shift. The representation given through $\hat{X}^{L}$ and $\hat{P}^{L}$ of Equation (7) on $\mathcal{K}$ directly extends to arbitrary functions $\alpha(p, x)$ and coincides with the $\star$ product structure with $\hat{\alpha}|\phi\rangle$ described in $\mathcal{K}$ as $\hat{\alpha}^{L} \phi=\alpha \star \phi$ and $\alpha \star \beta \star=(\alpha \star \beta) \star$ as $\alpha \star(\beta \star \phi)=(\alpha \star \beta) \star \phi$. It is the left regular representation of the functional algebra on itself, which can be extended further to all of $S^{\prime}(\Pi)$. One can even associate the wavefunction $\phi$ with a $\hat{\phi}^{L}=\phi \star$ operator, though the latter does not correspond to a physical observable. It remains to be seen if the operator has any particular physical meaning. Looking at a real wavefunction, or $|\phi|$, makes more sense as the absolute phase of a quantum state has no physical meaning anyway. $|\phi| \star$ makes a legitimate physical observable. We are interested only in applying all these mathematical results to the Gelfand triple $\mathcal{S}<\mathcal{K}<\mathcal{S}^{\prime}$ and that is the background on which the explicit results concerning the states are to be understood. We will see at the end that $\mathcal{K}$ is essentially the left ideal of $L^{2}(\Pi)$ that carries an irreducible representation of the Moyal algebra.

The Wigner functions that describe states, pure or mixed, are to be given in terms of functions $\rho$ of the density operator $\hat{\rho}$. For a pure state $|\phi\rangle$, the latter is denoted by $\hat{\rho}_{\phi} \equiv|\phi\rangle\langle\phi|$. Two different pure states give $\hat{\rho}_{\phi \phi^{\prime}} \equiv\left|\phi^{\prime}\right\rangle\langle\phi|$, with a non-diagonal Wigner function given as $\rho_{\phi \phi^{\prime}}=2^{n} \operatorname{Tr}\left[\hat{\rho}_{\phi \phi^{\prime}} \Delta_{p, x}^{\dagger}\right]$. Focusing on the set of basis coherent states, one can check that actually $F\left[\phi_{a}\right]=\phi_{a}$ and $F\left[\bar{\phi}_{a}\right]=\bar{\phi}_{-a}$. For $\hat{\rho}_{a b} \equiv\left|p_{b}, x_{b}\right\rangle\left\langle p_{a}, x_{a}\right|$, we have, with Equation (23),

$$
\rho_{a b}(p, x)=2^{2 n} \phi_{b} \circ \bar{\phi}_{a}=2^{2 n} F\left[\phi_{b}\right] \star \bar{\phi}_{a}=2^{2 n} \phi_{b} \star \bar{\phi}_{a} .
$$

Explicitly,

$$
\rho_{a b}(p, x)=2^{n} \mathrm{e}^{i\left(p_{a} x_{b}-x_{a} p_{b}\right)} \mathrm{e}^{i\left(p_{b} x-x_{b} p\right)} \mathrm{e}^{-i\left(p_{a} x-x_{a} p\right)} \mathrm{e}^{-\frac{\left(p-p_{a}-p_{b}\right)^{2}+\left(x-x_{a}-x_{b}\right)^{2}}{2}},
$$

which for $b=a$ reduces to

$$
\rho_{a}(p, x)=2^{n} \mathrm{e}^{-\frac{\left(p-2 p_{a}\right)^{2}+\left(x-2 x_{a}\right)^{2}}{2}} .
$$

The latter is the Wigner function for the coherent state $\left|p_{a}, x_{a}\right\rangle$, a Gaussian of unit width centered at $\left(2 p_{a}, 2 x_{a}\right)$ the point with coordinates exactly at the expectation values. We can then obtain

$$
\begin{gathered}
\operatorname{Tr}\left[\hat{\alpha}^{L}\right]=\frac{1}{\pi^{n}} \int \mathrm{d} p_{a} \mathrm{~d} x_{a} \frac{1}{\pi^{n}} \int \mathrm{d} p \mathrm{~d} x \bar{\phi}_{a}\left(\alpha \star \phi_{a}\right) \\
=\frac{1}{2^{n}(2 \pi)^{n}} \int \mathrm{d} p \mathrm{~d} x \alpha \frac{1}{\pi^{n}} \int \mathrm{d} p_{a} \mathrm{~d} x_{a} \rho_{a}=\frac{1}{2^{n}(2 \pi)^{n}} \int \mathrm{d} p \mathrm{~d} x \alpha,
\end{gathered}
$$


in which we have used the associativity and trace properties of the star product. This result corresponds to the standard trace expression for $\alpha$, or rather $\alpha \star$ $(\alpha=\alpha \star 1)$. We also denote this by $\operatorname{Tr}[\alpha]$ for simplicity. From a transition amplitude, we have

$$
\frac{1}{\pi^{n}} \int \mathrm{d} p \mathrm{~d} x \alpha\left(\phi^{\prime} \star \bar{\phi}\right)=\operatorname{Tr}\left[\alpha \star \rho_{\phi \phi^{\prime}} \star\right]=\frac{1}{2^{n}} \frac{1}{(2 \pi)^{n}} \int \mathrm{d} p \mathrm{~d} x \alpha \rho_{\phi \phi^{\prime}}
$$

The latter result includes as special cases the standard $\langle\phi|\hat{\alpha}| \phi\rangle=\operatorname{Tr}\left[\alpha \rho_{\phi}\right]$ and the somewhat strange looking $\frac{1}{\pi^{n}} \int \mathrm{d} p \mathrm{~d} x \bar{\phi} \phi=\frac{1}{2^{n}} \frac{1}{(2 \pi)^{n}} \int \mathrm{d} p \mathrm{~d} x \rho_{\phi}{ }^{5}$. Actually, we have

$$
\rho_{\phi \phi^{\prime}}=2^{2 n} \phi^{\prime} \circ \bar{\phi}=2^{2 n} F\left[\phi^{\prime}\right] \star \bar{\phi}=2^{2 n} \phi^{\prime} \star \bar{\phi},
$$

which may be considered as following from Equation (32), since any state (wavefunction) can be taken as a linear combination of the $\phi_{a}$ basis states. One can further check explicitly that $\operatorname{Tr}\left[\rho_{a}^{2}\right]=\operatorname{Tr}\left[\rho_{a}\right]=1, \rho_{a} \star \rho_{a}=\rho_{a}$, and $\rho_{a} \star \phi_{a}=\phi_{a}$ for the functions $\rho_{a}$ and $\phi_{a}$. Another interesting result is

$$
[\alpha \star \phi]\left(p_{a}, x_{a}\right)=\frac{1}{\pi^{n}} \int \mathrm{d} p_{b} \mathrm{~d} x_{b} \operatorname{Tr}\left[\alpha \rho_{a b}\right] \phi\left(p_{b}, x_{b}\right),
$$

which is the key result in Ref. [37] giving, together with Equation (33), the explicit integral kernel of the operator $\alpha \star$; more specifically we have

$$
\phi\left(p_{a}, x_{a}\right)=\frac{1}{\pi^{n}} \int \mathrm{d} p_{b} \mathrm{~d} x_{b} \operatorname{Tr}\left[\rho_{a b}\right] \phi\left(p_{b}, x_{b}\right) .
$$

The set of $\rho_{a b}$ spans the Hilbert space $L^{2}(\Pi)$, or equivalently the set of $\rho_{a b} \star$ spans $\mathcal{T}_{2}(\mathcal{K})$, which is the space of Hilbert-Schmidt operators, with the inner product $\langle\langle\alpha \mid \beta\rangle\rangle=\operatorname{Tr}[\bar{\alpha} \beta] \cdot\left[L^{2}(\Pi),{ }^{-}\right]$is a generalized Hilbert algebra [42] and $\left[S(\Pi),{ }^{-}\right]$a subalgebra.

In the algebraic formulation on $S(\Pi)<L^{2}(\Pi)<S^{\prime}(\Pi)$, the (normalized) states are to be defined by the positive and normalized functionals on $\mathcal{L}^{+}(S(\Pi))$ given by $\omega_{\rho}(\alpha)=\operatorname{Tr}[\alpha \rho]$; hence, essentially the set of density operator functions $\rho$ (we will use the term density matrix for such a function below). Each $\rho_{\phi} \star$ is a projection operator, and the one-dimensional projec${ }^{5}$ It is interesting to see the consistency of this result for the explicit case of a coherent state $\phi_{a}$. The normalization condition for a wavefunction in $\mathcal{K}$ can be written in the form

$$
\begin{aligned}
1 & =\frac{1}{\pi^{n}} \int \mathrm{d} p \mathrm{~d} x \bar{\phi} \phi=\frac{1}{2^{n}} \frac{1}{(2 \pi)^{n}} \int \mathrm{d}(2 p) \mathrm{d}(2 x) \mathrm{e}^{-\frac{1}{2}\left[2\left(p-p_{a}\right)^{2}+2\left(x-x_{a}\right)^{2}\right]} \\
& \left.=\frac{1}{2^{n}} \frac{1}{(2 \pi)^{n}} \int \mathrm{d} \tilde{p}^{s} \mathrm{~d} \tilde{x}^{s} \mathrm{e}^{-\frac{1}{8}\left[2\left(\tilde{p}^{s}-2 p_{a}\right)^{2}+2\left(\tilde{x}^{s}-2 x_{a}\right)^{2}\right]}\right]
\end{aligned}
$$

to be compared with

$$
1=\frac{1}{2^{n}} \frac{1}{(2 \pi)^{n}} \int \mathrm{d} p \mathrm{~d} x \rho_{a}=\frac{1}{(2 \pi)^{n}} \int \mathrm{d} p \mathrm{~d} x \mathrm{e}^{-\frac{1}{2}\left[\left(p-2 p_{a}\right)^{2}+\left(x-2 x_{a}\right)^{2}\right]} .
$$

In terms of the new variables we have $\phi_{a}\left(\tilde{p}^{s}, \tilde{x}^{s}\right)=\mathrm{e}^{\frac{i}{2}\left(p_{a} \tilde{x}^{s}-x_{a} \tilde{p}^{s}\right)} \mathrm{e}^{-\frac{1}{8}\left[\left(\tilde{p}^{s}-2 p_{a}\right)^{2}+\left(\tilde{x}^{s}-2 x_{a}\right)^{2}\right]}$, a Gaussian centered at the expectation values $\left(2 p_{a}, 2 x_{a}\right)$ with width $\frac{1}{2}$. 
tions correspond to pure states $\omega_{\phi} \equiv \omega_{\rho_{\phi}}$. The Hilbert space is to be constructed as the completion of the quotient $\mathcal{I}_{\mathcal{K}} / \mathcal{I}_{o}$ of closed left ideals with a (pre-)inner product on $\mathcal{I}_{\mathcal{K}}$ through an $\omega_{\phi}$ to which $\mathcal{I}_{o}$ is the kernel [26] [38] [39]. An obvious choice here is the Hilbert space $\mathcal{I}_{\mathcal{K}}=\left\{\alpha \star \rho_{o}: \alpha \in L^{2}(\Pi)\right\}$ and $\mathcal{I}_{o}$ of $\left[L^{2}(\Pi), \star^{-},\right]$with the inherited inner product $\langle\alpha \mid \beta\rangle_{\omega_{o}} \equiv\left\langle\left\langle\alpha \star \rho_{o} \mid \beta \star \rho_{o}\right\rangle\right\rangle=\omega_{o}(\bar{\alpha} \star \beta)$ for which $\mathcal{I}_{o}=\{0\}$. Note that the inner product is exactly the same as $\left\langle\hat{\alpha} \phi_{o} \mid \hat{\beta} \phi_{o}\right\rangle$ illustrating that the Hilbert space is equivalent to $\mathcal{H}$ or $\mathcal{K}$. In fact, $\rho_{o}=2^{n} \phi_{o}$; hence $\mathcal{K}$ and $\mathcal{I}_{\mathcal{K}}$ differ only in normalizations. $\mathcal{I}_{\mathcal{K}}$ is invariant under the action of the Moyal algebra $\mathcal{M}^{\prime}$ and as a representation it is a faithful and irreducible one, which matches with it being irreducible as a representation of the Heisenberg-Weyl symmetry. After all, the algebra of observables is to include the enveloping algebra of the latter. Any particular wavefunction $\phi$ of $\mathcal{K}$ can be used to give a representation through $\omega_{\phi}$ which are all unitary equivalent. So, we come full circle to the representation on $\mathcal{K}$ giving an explicit illustration of the more abstract algebraic language through the WWGM framework.

\section{Lie Algebra Contraction Limit}

Consider the contraction [40] [41] of the Lie algebra for the Heisenberg-Weyl subgroup of the full relativity symmetry given by the $k \rightarrow \infty$ limit with the rescaled generators

$$
X_{i}^{c}=\frac{\sqrt{\hbar}}{k} X_{i} \text { and } P_{i}^{c}=\frac{\sqrt{\hbar}}{k} P_{i} .
$$

We have $\left[X_{i}^{c}, P_{j}^{c}\right]=i \frac{2 \hbar}{k^{2}} \delta_{i j} I \rightarrow 0(\hbar)$. Here, $k$ is a pure numerical parameter while $\hbar$ is Planck's constant, which is needed to allow $X_{i}^{c}$ and $P_{j}^{c}$ to take on independent physical units, such as the usual classical units. One can take the parameter $k$ here as $\sqrt{2}$ to give the standard form of the commutator $\left[X_{i}^{c}, P_{j}^{c}\right]=i \hbar I$ and think of the contraction limit as the $\hbar \rightarrow 0$ limit, i.e. as the classical limit is usually envisaged. However, as discussed in Ref. [4], naively taking $\hbar$ to zero everywhere in the theory written with $\hbar$ carrying nontrivial physical units is not the right thing to do; case examples can be seen below. On the other hand, the contraction limit is of course independent of the contraction parameter used, and the physical units for $X_{i}^{c}$ and $P_{i}^{c}$ differ from those of $X_{i}$ and $P_{i}$ by the same factor of the physical unit (that of $\sqrt{\hbar}$ ), even in the limit. To keep track of things carefully, in a way that enables the reader to see expressions in their familiar forms with a nonzero $\hbar$ as well as the contraction limit results, we keep $\hbar$ and $k$ separate in this section. As we mentioned above, substituting $\sqrt{2}$ for $k$ yields the familiar quantum expressions with their proper $\hbar$ dependence, and then we can interpret the naive choice of taking $\hbar \rightarrow 0$ (which can be interpreted in the classical system of units) as the classical limit; however, we instead take $k \rightarrow \infty$ as the appropriate choice for describing the classical limit in the symmetry (representation) contraction perspective. 
We first rewrite the Heisenberg-Weyl group element in the usual form

$$
\begin{aligned}
W\left(\breve{p}^{i}, \breve{x}^{i}, \theta\right)=\mathrm{e}^{\frac{i}{\hbar}\left(\breve{p}^{i} X_{i}^{c}-\breve{x}^{i} P_{i}^{c}+\theta \hbar I\right)}, \text { where } \\
\qquad \breve{p}^{i}=\sqrt{\hbar} k p^{i}, \text { and } \breve{x}^{i}=\sqrt{\hbar} k x^{i} .
\end{aligned}
$$

Here, $\hbar$ is introduced so that $\breve{p}^{i}$ and $\vec{x}^{i}$ carry the same units as $P_{i}^{c}$ and $X_{i}^{c}$, respectively. As for the invariant vector fields acting on the group manifold, we have

$$
\begin{aligned}
X^{c L} & =\frac{\breve{x}}{k^{2}} i \partial_{\theta}+i \hbar \partial_{\breve{p}} \rightarrow i \hbar \partial_{\breve{p}}, \\
P^{c L} & =\frac{\breve{p}}{k^{2}} i \partial_{\theta}-i \hbar \partial_{\breve{x}} \rightarrow-i \hbar \partial_{\breve{x}} .
\end{aligned}
$$

Note that the contraction limits of these vector fields, as given above, carry a factor of $\hbar$. This is needed in order to have the correct physical units; the dropping out of the terms $i \partial_{\theta}$ is to be expected, as the central charge $I$ fully decouples from the rest of the algebra. In particular, observe that, had we replaced $k^{2}$ by 2 , taking the naive $\hbar \rightarrow 0$ limit would yield an incorrect result with only $i \partial_{\theta}$ in the classical limit.

To trace the contraction of the coherent state representation, simply relabeling the set of $|p, x\rangle$ states by $|\breve{p}, \breve{x}\rangle=U(\breve{p}, \breve{x})|0\rangle$ with $U(\breve{p}, \breve{x})=\mathrm{e}^{\frac{i}{\hbar}\left(\breve{p}^{i} \hat{X}_{i}^{c}-\breve{x}^{i} \hat{P}_{i}^{c}\right)}$ is not the right thing to do. It should be emphasized that the basic idea for taking the classical approximation as the symmetry contraction limit is to take the original representation which describes the quantum physics to the required limit rather than directly building the classical physics description from the contracted symmetry. We will see that the contraction of the representation does indeed give a representation of the contracted symmetry though. The essence of the coherent state representation is to have the states labeled essentially by their (finite) expectation values. However, we have $x_{i}^{s} \equiv\left\langle\breve{p}, \breve{x}\left|\hat{X}_{i}^{c}\right| \breve{p}, \breve{x}\right\rangle=\frac{2}{k^{2}} \breve{x}_{i}$ and similarly $p_{i}^{s}=\frac{2}{k^{2}} \breve{p}_{i}$. Let us instead take $\left|p^{s}, x^{s}\right\rangle$ and note that

$$
p^{s}=\frac{2 \sqrt{\hbar}}{k} p, \text { and } x^{s}=\frac{2 \sqrt{\hbar}}{k} x .
$$

On the Hilbert space $\mathcal{K}$ of wavefunctions, we then have

$$
\begin{aligned}
& \hat{X}^{c L}=\frac{x^{s}}{2}+i \frac{2 \hbar}{k^{2}} \partial_{p^{s}} \rightarrow \frac{x^{s}}{2}, \\
& \hat{P}^{c L}=\frac{p^{s}}{2}-i \frac{2 \hbar}{k^{2}} \partial_{x^{s}} \rightarrow \frac{p^{s}}{2} .
\end{aligned}
$$

The difference between these results and those of Equation (43) is remarkable. The overlap between the different coherent states $\left\langle p^{s^{\prime}}, x^{s^{\prime}} \mid p^{s}, x^{s}\right\rangle$ can be obtained easily from Equation (9). As discussed in Ref. [4], it vanishes at the contraction limit and $\mathcal{H}$, as a representation for the observable algebra, which is to be interpreted as functions of $\hat{X}^{c}$ and $\hat{P}^{c}$ reducing to a simple sum of the 
one-dimensional spaces for each $\left|p^{s}, x^{s}\right\rangle$. On $\mathcal{K}$, the coherent state wavefunctions $\phi_{a}\left(p^{s}, x^{s}\right)$ are each, apart from a phase factor, a Gaussian with width $\frac{\sqrt{2 \hbar}}{k}$, and hence collapse to the delta function $\delta_{a}\left(p^{s}, x^{s}\right)$ in the limit. The classical coset space picture [4] of the Newtonian phase space can be considered as having been retrieved. Sticking to the Hilbert space picture,

$\phi_{a}\left(p^{s}, x^{s}\right) \rightarrow \delta_{a}\left(p^{s}, x^{s}\right)$ makes $\mathcal{K}$ the whole of $L^{2}(\Pi) \equiv L^{2}\left(\Pi^{s}\right)$, which is the Koopman Hilbert space for classical mechanics [36]. A word of caution is warranted on the integration measure. The normalization condition of $\phi_{0}\left(p^{s}, x^{s}\right)$ reads

$$
\left(\frac{k^{2}}{4 \pi \hbar}\right)^{n} \int \mathrm{d} p^{s} \mathrm{~d} x^{s} \mathrm{e}^{-\frac{k^{2}}{4 \hbar}\left[\left(p^{s}\right)^{2}+\left(x^{s}\right)^{2}\right]}=1
$$

the measure of which apparently diverges at the $k \rightarrow \infty$ limit. Similarly, $\hat{I}=\frac{1}{\pi^{n}} \mathrm{~d} p \mathrm{~d} x|p, x\rangle\left\langle p, x\left|=\left(\frac{k^{2}}{4 \hbar \pi}\right)^{n} \int \mathrm{d} p^{s} \mathrm{~d} x^{s}\right| p^{s}, x^{s}\right\rangle\left\langle p^{s}, x^{s}\right|$. The delta function limit has the measure $\frac{1}{(2 \pi \hbar)^{n}} \int \mathrm{d} p^{s} \mathrm{~d} x^{s}$ instead. As the $k$-dependence drops at the $k \rightarrow \infty$ limit, we need the $\hbar$ to fix the physical units. The set of $\phi_{a}\left(p^{s}, x^{s}\right)$, then as $\delta_{a}$, as a basis for $\mathcal{K}$ is maintained. However, a generic function as a linear combination of $\phi_{a}\left(p^{s}, x^{s}\right)$ loses the status of being a physical state at least as far as pure states are concerned. The basis coherent states, or rather their classical limit as given by $\phi_{a}\left(p^{s}, x^{s}\right)$ (or simply points $\left(p^{s}, x^{s}\right)$ of the familiar classical phase spaces), are the only pure states.

Next, we look at all of the above integral transformations in order to track the Weyl correspondence at the contraction limit. Here, it is not so clear whether we should use the group parameters $\breve{p}$ and $\breve{x}$ or the coherent state parameters. In fact, both work. Note that in the discussion of the coherent state above, in view of things in the last section, it is better to use $\left|p^{c}, x^{c}\right\rangle$ with

$$
p^{c}=\frac{p^{s}}{2}=\frac{\sqrt{\hbar}}{k} p \text {, and } x^{c}=\frac{x^{s}}{2}=\frac{\sqrt{\hbar}}{k} x
$$

instead, especially as that gives the ratio between the old and new parameters as the same as that between the operators [cf. Equation (41)]. $p^{s}$ and $x^{s}$ were mostly used there for easy reading of familiar results (at $k^{2}=2$ ). Explicitly, we have

$$
\begin{aligned}
& \hat{X}^{c L}=x^{c}+i \frac{1}{k^{2}} \partial_{p^{c}} \rightarrow x^{c}, \\
& \hat{P}^{c L}=p^{c}-i \frac{1}{k^{2}} \partial_{x^{c}} \rightarrow p^{c},
\end{aligned}
$$

giving a nice contraction limit. That is of course nothing more than a convenient choice of convention, one we adopted in the beginning through labeling the coherent states by half their expectation values. In terms of the new parameters, we have 


$$
\Omega^{(L)}[\alpha]=\left(\frac{k^{2}}{2 \pi \hbar}\right)^{n} \int \mathrm{d} p^{c} \mathrm{~d} x^{c} \alpha\left(p^{c}, x^{c}\right) U^{(L)}\left(p^{c}, x^{c}\right),
$$

and

$$
F[\alpha]=\left(\frac{k^{2}}{2 \pi \hbar}\right)^{n} \int \mathrm{d} p^{c} \mathrm{~d} x^{c} \alpha\left(p^{c}, x^{c}\right) \mathrm{e}^{\frac{i k^{2}}{\hbar}\left(p^{c} x^{c^{\prime}}-x^{c} p^{c^{\prime}}\right)} .
$$

Note that-despite the factor of $k^{2 n}$ in front-the latter two expressions are $k$-independent. The factor of $k^{2 n}$ cancels after integration; note also that

$$
\begin{aligned}
U^{(L)}\left(p^{c}, x^{c}\right) & =\mathrm{e}^{\frac{i k^{2}}{\hbar}\left[p^{c} \hat{X}^{(L)}-x^{c} \hat{P}^{(L)}\right]} . \text { It follows that } \\
\Delta^{(L)}[\alpha] & =\Omega^{(L)}\left[F^{-1}[\alpha]\right] \\
& =\left(\frac{k^{2}}{2 \pi \hbar}\right)^{2 n} \int \mathrm{d} p^{c} \mathrm{~d} x^{c} \mathrm{~d} p^{c^{\prime}} \mathrm{d} x^{c^{\prime}} \alpha\left(p^{c}, x^{c}\right) \mathrm{e}^{\frac{i k^{2}}{\hbar}\left(x^{c} p^{c}-p^{c} x^{c}\right)} U^{(L)}\left(p^{c^{\prime}}, x^{c^{\prime}}\right) \\
& =\left(\frac{k^{2}}{2 \pi \hbar}\right)^{n} \int \mathrm{d} p^{c} \mathrm{~d} x^{c} \alpha\left(p^{c}, x^{c}\right) \Delta_{p^{c}, x^{c}}^{(L)},
\end{aligned}
$$

where we have

$$
\Delta_{p, x}^{(L)}=\left(\frac{k^{2}}{2 \pi \hbar}\right)^{n} \int \mathrm{d} p^{c^{\prime}} \mathrm{d} x^{c^{\prime}} \mathrm{e}^{\frac{i k^{2}}{\hbar}\left(x^{c^{\prime}} p^{c}-p^{c^{\prime}} x^{c}\right)} U^{(L)}\left(p^{c^{\prime}}, x^{c^{\prime}}\right) .
$$

The Weyl correspondence is obviously maintained ${ }^{6}$; explicitly, we have $\Delta\left[\alpha\left(p^{c}, x^{c}\right)\right]=\alpha\left(\hat{P}^{c}, \hat{X}^{c}\right)$ and $\Delta^{L}\left[\alpha\left(p^{c}, x^{c}\right)\right]=\alpha\left(\hat{P}^{c L}, \hat{X}^{c L}\right)$. Analogous trace formulas for the inversion of the transforms work as well. With the original 。 with $\Omega^{(L)}$ written in terms of the parameters $p^{c}$ and $x^{c}$, we have the Moyal star product given by

$$
\star^{c} \sim \exp \left[\frac{-i \hbar}{k^{2}}\left(\bar{\partial}_{p^{c}} \vec{\partial}_{x^{c}}-\bar{\partial}_{x^{c}} \vec{\partial}_{p^{c}}\right)\right],
$$

${ }^{6}$ One may also consider

$$
\Omega^{\alpha(L)}[\alpha] \equiv \frac{1}{(2 \pi \hbar)^{n}} \int \mathrm{d} \breve{p} \mathrm{~d} \breve{x} \alpha(\breve{p}, \breve{x}) U^{(L)}(\breve{p}, \breve{x}),
$$

and

$$
F^{c}[\alpha] \equiv \frac{1}{(2 \pi \hbar)^{n}} \int \mathrm{d} \check{p} \mathrm{~d} \check{x} \alpha(\breve{p}, \breve{x}) \mathrm{e}^{\frac{i}{\hbar}\left(\bar{h} \bar{x}^{c}-\bar{p} \bar{p}^{c}\right)} .
$$

Actually, we have $\Omega^{\alpha(L)}[\alpha]=k^{2 n} \Omega^{(L)}[\alpha]$ and $F^{c}[\alpha]=k^{2 n} F[\alpha]$ formally $\left(F^{c-1} \neq F^{c}\right)$. It follows that

$$
\begin{aligned}
\Delta^{(L)}[\alpha] & =\Omega^{(L)}\left[F^{c-1}[\alpha]\right] \\
& =\frac{1}{(2 \pi \hbar)^{2 n}} \int \mathrm{d} p^{c} \mathrm{~d} x^{c} \mathrm{~d} \breve{p} \mathrm{~d} \breve{x} \alpha\left(p^{c}, x^{c}\right) \mathrm{e}^{\frac{i}{\hbar}\left(\bar{p} \bar{p}^{c}-\bar{p} x^{c}\right)} U^{(L)}(\breve{p}, \breve{x}) \\
& =\frac{1}{(2 \pi \hbar)^{n}} \int \mathrm{d} p^{c} \mathrm{~d} x^{c} \alpha\left(p^{c}, x^{c}\right) \Delta_{p^{c}, x^{c}}^{(L)},
\end{aligned}
$$

with $\Delta_{p^{\prime}, x^{c}}^{(L)}=\frac{1}{(2 \pi \hbar)^{n}} \int \mathrm{d} \breve{p} \mathrm{~d} \breve{\mathrm{e}^{\frac{i}{\hbar}}(\bar{x} p-\bar{x})} U^{(L)}(\breve{p}, \breve{x})\left[=k^{2 n} \Delta_{p, x}^{(L)}\right]$ The twisted convolution required to maintain $\Omega^{(L)}\left[\alpha 0^{c} \beta\right]=\Omega^{\alpha(L)}[\alpha] \Omega^{(L)}[\beta]$ is simply given formally by $k^{2 n} \alpha \circ \beta$, i.e.

$$
\alpha 0^{c} \beta(\breve{p}, \breve{x})=\frac{1}{(2 \pi \hbar)^{n}} \mathrm{~d} \breve{p}^{\prime} \mathrm{d} \breve{x}^{\prime} \alpha\left(\breve{p}^{\prime}, \breve{x}^{\prime}\right) \beta\left(\breve{p}-\breve{p}^{\prime}, \breve{x}-\breve{x}^{\prime}\right) \mathrm{e}^{\frac{i}{k^{2} \hbar}\left(\bar{x}^{\prime}-\breve{p}^{\prime}\right)} .
$$


which is the same old expression written in terms of the new parameters serving as arguments for the (observable) functions, though we denote it by $\star^{c}$ for convenience of later referral. The star product reduces to the simple product in the $k \rightarrow \infty$ limit, as it should. The equation $M_{\star}\left[\alpha\left(p^{c}, x^{c}\right)\right]=\alpha\left(p^{c}, x^{c}\right) \star^{c}$ is maintained for all finite $k$ and at the $k \rightarrow \infty$ limit where the $M_{\star}$ becomes the simple multiplication of the then classical observable $\alpha\left(p^{c}, x^{c}\right)$. The suggestive notation of the multiplicative operator $M\left[\alpha\left(p^{c}, x^{c}\right)\right]$, or simply $M_{\alpha}$, is standard for an operator on the $L^{2}(\Pi)$ Hilbert space representing classical observables in the Koopman-von Neumann formulation of classical mechanics [36].

The coherent state Wigner function is

$$
\rho_{a}\left(p^{c}, x^{c}\right)=2^{n} \mathrm{e}^{-\frac{k^{2}\left(p^{c}-2 p_{a}^{c}\right)^{2}-\left(x^{c}-2 x_{a}^{c}\right)^{2}}{2}},
$$

with width $\frac{\sqrt{\hbar}}{k}$, and it reduces to a delta function as $k \rightarrow \infty$. For the classical case, any "density matrix" $\rho(p, x)$ beyond the delta functions are to be interpreted as statistical distributions; hence they are mixed states. A generic $\rho(p, x)$ has the form

$$
\rho(p, x)=\sum_{m} c_{m} \rho_{\phi_{m}}(p . x)=2^{2 n} \sum_{m} c_{m}\left[\phi_{m} \star \bar{\phi}_{m}\right](p . x)
$$

for pure states $\phi_{m}(p . x) \in \mathcal{K}$, and $c_{m}$ are positive real numbers with $\sum_{m} c_{m}=1$. Therefore, we have

$$
\rho\left(p^{c}, x^{c}\right)=2^{2 n} \sum_{m} c_{m}\left[\phi_{m} \star^{c} \bar{\phi}_{m}\right]\left(p^{c}, x^{c}\right) \rightarrow 2^{2 n} \sum_{m} c_{m}\left|\phi_{m}\left(p^{c}, x^{c}\right)\right|^{2}
$$

as $k \rightarrow \infty$. To be more rigorous, one would have to introduce a rescaled/renormalized $\rho^{c}$ for the limiting classical density matrix as the conventional distribution to describe the classical statistical state through a bounded $\rho^{c}\left(p^{c}, x^{c}\right)=\lim _{k \rightarrow \infty} k^{2 n} \rho\left(p^{c}, x^{c}\right)$ in order to maintain $\frac{1}{(2 \pi \hbar)^{n}} \int \rho^{c} \mathrm{~d} p^{c} \mathrm{~d} x^{c}=1$. The classical wavefunction $\phi^{c}$, satisfying $\left|\phi^{c}\right|^{2} \equiv \rho^{c}$, for the Koopman-von Neumann formulation may then be introduced. It is important to emphasize that a function $\phi^{c}$ describes a mixed state. Only the limiting distributions of the delta functions describes the (classical) pure states.

An even better formal picture of the classical limit, which is also particularly useful for the description of dynamics and symmetries below, is offered by the notion of a Tomita representation [42] as presented in Ref. [43]. Note that the representation is not an irreducible one-an aspect that fits the Koopman-von Neumann formulation well. We present only a specific description based on the wavefunctions $\phi(p, x)$, although also using the $|\phi\rangle$ notation when it is more illustrative and convenient. Consider the Hilbert space $\tilde{\mathcal{K}}$ of square ket vectors $\mid \alpha]$ defined as the span of all $\left.\mid \phi^{\prime} \phi\right] \equiv \rho_{\phi \phi^{\prime}}$ with the inner product given by

$$
\left[\psi^{\prime} \psi \mid \phi^{\prime} \phi\right]=\operatorname{Tr}\left[\bar{\rho}_{\psi \psi^{\prime}} \rho_{\phi \phi^{\prime}}\right] \text {. }
$$


$\tilde{\mathcal{K}}$ is thus a tensor product of $\mathcal{K}$ with itself. It is essentially the space of Hilbert-Schmidt operators on $\mathcal{K}$, and can be thought of as the span of all $\rho_{a b}$. A vector $\mid \alpha]$ corresponds to $\alpha \in L^{2}(\Pi)$ or the Hilbert-Schmidt operator $\alpha \star$ on $\mathcal{K}$ and we can identify it with $|\alpha\rangle\rangle$, introduced towards the end of the last section. That is, within our formulation, $\mathcal{K}$ can be identified as $L^{2}(\Pi)$. Next, one can introduce the conjugation $J$ as an antiunitary operator with the properties:

$$
\begin{aligned}
& \text { (i) } \left.\left.J_{c} \mid \alpha\right]=\bar{c} J \mid \alpha\right] \quad \forall c \in \mathbb{C}, \\
& \text { (ii) }\left[\alpha\left|J^{\dagger} J\right| \gamma\right]=[\gamma \mid \alpha], \\
& \text { (iii) } J^{2}=I .
\end{aligned}
$$

State vectors in $\tilde{\mathcal{K}}$ are introduced as vectors in a self-dual cone $\tilde{\mathcal{D}}$ of with real and positive inner products. The vectors correspond to the mixed states. They satisfy $J \mid \rho]=\mid \rho] \equiv \rho$. For any operator $\alpha \star$ on $\mathcal{K}$, we have an operator $(\alpha \star)_{T}$ on $\tilde{\mathcal{K}}$ defined by

$$
\left.\left.(\alpha \star)_{T} \mid \phi^{\prime} \phi\right] \equiv \mid\left(\alpha \star \phi^{\prime}\right) \phi\right]=\rho_{\phi\left(\alpha \star \phi^{\prime}\right)}=\alpha \star \rho_{\phi \phi^{\prime}},
$$

as $\rho_{\phi \phi^{\prime}}=2^{2 n} \phi^{\prime} \star \bar{\phi}$. We have $\left.\left.J \mid \phi^{\prime} \phi\right]=\mid \phi \phi^{\prime}\right]$, hence $J(\alpha \star)_{T} J \rho_{\phi \phi^{\prime}}=\rho_{(\alpha \star \phi) \phi^{\prime}}$. Note that $(\alpha \star)_{T}$ and $J(\alpha \star)_{T} J$ act on different parts of the tensor product; explicitly $(\alpha \star)_{T}=(\alpha \star) \otimes 1$ and $J(\alpha \star)_{T} J=1 \otimes(\alpha \star)$. More explicitly, we have

$$
J \beta=\bar{\beta},
$$

and

$$
\begin{gathered}
(\alpha \star)_{T} \beta=\alpha \star \beta, \\
J(\alpha \star)_{T} J \beta=\beta \star \bar{\alpha},
\end{gathered}
$$

where we have extended the results for $\rho_{\phi \phi^{\prime}}$ to a generic $\beta \in \tilde{\mathcal{K}}$, and utilized some algebraic properties of the star product, including $\overline{\alpha \star \beta}=\bar{\beta} \star \bar{\alpha}$. A pure state is then represented by $\left.\left.\mid \rho_{\phi}\right] \equiv \mid \phi \phi\right]$ and we have $\left[\rho_{\psi} \mid \rho_{\phi}\right]=\operatorname{Tr}\left[\rho_{\psi} \rho_{\phi}\right]=|\langle\psi \mid \phi\rangle|^{2}$; hence $\left.\mid \rho_{\phi}\right]$ is normalized. The expectation value $\left[\rho_{\phi}\left|(\alpha \star)_{T}\right| \rho_{\phi}\right]$ for a pure state is the same as on $\mathcal{K}$, i.e. equal to $\langle\phi|\hat{\alpha}| \phi\rangle$ and $\operatorname{Tr}\left[\alpha \rho_{\phi}\right]$; and for a mixed state $\left[\rho\left|(\alpha \star)_{T}\right| \rho\right]=\operatorname{Tr}[\alpha \rho]$. Transition probabilities are given by $\left[\rho_{\psi}\left|(\alpha \star)_{T} J(\alpha \star)_{T} J\right| \rho_{\phi}\right]=|\langle\psi \mid \hat{\alpha} \phi\rangle|^{2}=\left(\operatorname{Tr}\left[\alpha \rho_{\psi \phi}\right]\right)^{2}$. Note that $\left.\mid \rho\right] \in \tilde{\mathcal{D}}$ always gives $\left.\left|(\alpha \star)_{T} J(\alpha \star)_{T} J\right| \rho\right] \in \tilde{\mathcal{D}}$. Observables on $\tilde{\mathcal{D}}$ are to be taken from those within the $(\alpha \star)_{T}$ and $J(\alpha \star)_{T} J$ set. Other vectors outside $\tilde{\mathcal{D}}$ and operators on $\tilde{\mathcal{K}}$ beyond this collection are not of much interest.

As $\tilde{\mathcal{K}}$ is essentially the space of Hilbert-Schmidt operators on $\mathcal{K}$, the classical picture from the contraction limit of the latter as a representation of the 
Heisenberg-Weyl symmetry obviously maintains the basic notion of the Hilbert space as $L^{2}(\Pi)$ to be coordinated by the classical phase space variables $\left(p^{c}, x^{c}\right)$, though a renormalization may again be necessary to trace them from the explicit original quantum $(\alpha \star)$. The original $\tilde{\mathcal{D}}$ is really the real span of $\rho_{a}$ for the coherent state basis; hence it becomes the real span of the delta functions in the classical limit. That is to say, the set of classical "density matrices" fills the whole real part of $L^{2}(\Pi)$. Formally, $\tilde{\mathcal{D}}^{c}$ is simply the real part of $\tilde{\mathcal{K}}^{c}$. We can see further that $(\alpha \star)_{T} \rightarrow M_{\alpha}$ and $J(\alpha \star)_{T} J \rightarrow M_{\bar{\alpha}}$. More features of the classical picture obtained will be seen in Section 7 , where we discuss the description of dynamics.

\section{Description of Quantum Symmetries and Time Evolution}

The description of the quantum symmetries in connection with the WWGM formalism has been well presented in Ref. [46] [47], from which we summarize the basic features and give explicit details for applications to our framework with particular emphasis on the elements of the relativity symmetry. Hermitian operators, as physical observables, play the role of the symmetry generators giving rise to a group of unitary flow on the Hilbert space(s), as well as an isomorphic group of automorphisms on the set of pure state density operators. Here we only focus on $\mathcal{K}$ and the matching set of $\rho_{\phi}$, while extending from the latter to all of $\tilde{\mathcal{D}} \in \tilde{\mathcal{K}}$ in the language of the Tomita representation.

Firstly, we have on $\mathcal{K}$ symmetries as the group of unitary and antiunitary operators factored by its closed center of phase transformations. The isomorphic automorphism group $\operatorname{Aut}(\mathcal{P})$ of the set $\mathcal{P}$ of $\rho_{\phi}$ is characterized by the subgroup of the group of real unitary transformations $\mathcal{O}\left(\tilde{\mathcal{K}}_{R}\right)$ compatible with the star product, i.e. $\mu \in A u t(\mathcal{P})$ satisfies

$$
\mu(\alpha \star \beta)=\mu(\alpha) \star \mu(\beta)
$$

[or $\mu(\alpha \star \beta \star)=\mu(\alpha) \star \mu(\beta) \star$ ]. $\tilde{\mathcal{K}}_{R}$ is the real subspace of the Hilbert space of Hilbert-Schmidt operators. Symmetry groups represented as subgroups of $\operatorname{Aut}(\mathcal{P})$ can be considered. For a star-unitary transformation $U_{\star}$ on wavefunctions $\phi \in \mathcal{K}$, we have a real unitary operator on $\tilde{\mathcal{K}}_{R}$

$$
\tilde{U}_{\star} \alpha \star=\mu(\alpha) \star=U_{\star} \star \alpha \star \bar{U}_{\star} .
$$

We write a generic one parameter group of such a (star-) unitary transformation in terms of real parameter $s$ as $U_{\star}(s) \star=\mathrm{e}^{\frac{-i s}{2} G_{s} \star}$ with $G_{s} \star$ as the generator. Note that the factor 2 is put in the place of $\hbar$, consistent with our choice of units ${ }^{7}$. For time translation, as a unitary transformation on $\mathcal{K}$, we have the Schrödinger equation of motion

$$
2 i \frac{\mathrm{d}}{\mathrm{d} t} \phi=G_{t} \star \phi .
$$

${ }^{7}$ For readers who find the factor of 2 difficult to appreciate, our results below in the next sectionespecially with the symmetry description in terms of the rescaled parameters in the usual units with an explicit $\hbar$-should make the full picture more transparent. 
In the Tomita representation picture, the unitary transformation $U_{\star}(s) \star$ on $\mathcal{K}$ gives a corresponding unitary transformation on $\tilde{\mathcal{K}}$ as the Hilbert space of square kets, which are here simply elements in $L^{2}(\Pi)$, given by [43]

$$
\tilde{U}_{\star}(s)=U_{\star}(s) J U_{\star}(s) J=\mathrm{e}^{\frac{-i s}{2}\left[\left(G_{s} \star\right)_{T}-J\left(G_{s} \star\right)_{T} J\right]} .
$$

From Equation (61), one can see that this is just a fancy restatement of Equation (63) above, with now an explicit form of $\tilde{U}_{\star}(s)$ as an operator in terms of the real generator function $G_{s}(p, x)$. Consider the generator $\tilde{G}_{s}$ as defined by $\tilde{U}_{\star}(s)=\mathrm{e}^{\frac{-i s}{2} \tilde{G}_{s}}$, we have

$$
\left.\left.\tilde{G}_{s} \rho \equiv \tilde{G}_{s} \mid \rho\right]=\left[\left(G_{s} \star\right)_{T}-J\left(G_{s} \star\right)_{T} J\right] \mid \rho\right]=G_{s} \star \rho-\rho \star G_{s}=\left\{G_{s}, \rho\right\}_{\star},
$$

where $\{\because \cdot\}_{\star}$ is the star product commutator, i.e. the Moyal bracket. Hence, with $\rho(s)=\tilde{U}_{\star}(s) \rho(s=0)$,

$$
\frac{\mathrm{d}}{\mathrm{d} s} \rho(s)=\frac{1}{2 i}\left\{G_{s}, \rho(s)\right\}_{\star} .
$$

The result is of course to be expected. When applied to the time translation as a unitary transformation, it gives exactly the Liouville equation of motion for a density matrix as the Schrödinger equation for the latter taken as a state in $\tilde{\mathcal{D}}$ with Hamiltonian operator $\tilde{\kappa}=\tilde{G}_{t} . G_{s}\left(p^{i}, x^{i}\right) \star=G_{s}\left(p_{i} \star, x_{i} \star\right)=G_{s}\left(\hat{P}_{i}^{L}, \hat{X}_{i}^{L}\right)$ is the operator that represents the (real) element $G_{s}\left(P_{i}, X_{i}\right)$ in the algebra of observables as well as $\mathcal{K} . \tilde{G}_{s}=\left\{G_{s},\right\}_{\star}$ then represents the algebra element on $\tilde{\mathcal{K}}$. It is interesting to note that by introducing the notation $\hat{G}_{s}^{L} \equiv G_{s}\left(p^{i}, x^{i}\right) \star$ as a left action, we have a corresponding right action $\hat{G}_{s}^{R}$ given by $\hat{G}_{s}^{R} \alpha=\alpha \star G_{s}$ with $\tilde{G}_{s}=\hat{G}_{s}^{L}-\hat{G}_{s}^{R}$. The explicit expression for the $\hat{G}_{s}^{R}$ action follows from

$$
\begin{aligned}
& \hat{X}_{i}^{R}=x_{i}-i \partial_{p^{i}}, \\
& \hat{P}_{i}^{R}=p_{i}+i \partial_{x^{i}} .
\end{aligned}
$$

These operators match to the right-invariant vector fields of the HeisenbergWeyl group as $\hat{X}^{L}$ and $\hat{P}^{L}$ corresponds to the left-invariant ones. When $G_{s}(p, x)$ is an order one or two polynomial in the variables, which covers the cases of interest here, $\tilde{G}_{s}$ has a very simple explicit form. Another important feature to note is that $\tilde{G}_{s}$ determines $G_{s}(p, x)$ only up to an additive constant. This is a consequence of the fact that the density matrix $\rho_{\phi}$ is insensitive to the phase of the pure state $\phi$. Constant functions in the observable algebra correspond to multiples of $\hat{I}$ on $\mathcal{H}$ which generates pure phase transformations, i.e. $G_{\theta}(p, x)=1$ and $\tilde{G}_{s}=0$.

Let us focus first on the observables $x \star$ and $p \star$ as symmetry generators on $\mathcal{K}$, and look at the corresponding transformations on $\tilde{\mathcal{D}}$. From Equation (8),

$$
U_{\star}(x) \star \phi\left(p^{\prime}, x^{\prime}\right)=\mathrm{e}^{\frac{-i x}{2}(-p \star)} \phi\left(p^{\prime}, x^{\prime}\right)=\phi\left(p^{\prime}, x^{\prime}+\frac{x}{2}\right) \mathrm{e}^{i x p^{\prime}},
$$




$$
U_{\star}(p) \star \phi\left(p^{\prime}, x^{\prime}\right)=\mathrm{e}^{\frac{-i p}{2}(x \star)} \phi\left(p^{\prime}, x^{\prime}\right)=\phi\left(p^{\prime}+\frac{p}{2}, x^{\prime}\right) \mathrm{e}^{-i p x^{\prime}},
$$

giving, in terms of explicit $x^{i}$ and $p^{i}$ parameters,

$$
\begin{aligned}
& G_{-x^{i}} \star=p_{i} \star, \quad \tilde{p}_{i}=\tilde{G}_{-x^{i}}=2 i \partial_{x^{i}}, \\
& G_{p^{i}} \star=x_{i} \star, \quad \tilde{x}_{i}=\tilde{G}_{p^{i}}=2 i \partial_{p^{i}} .
\end{aligned}
$$

The factors of 2 in the translations $U_{\star}(x) \star$ and $U_{\star}(p) \star$ may look somewhat suspicious at first sight. They are actually related to the fact that the arguments of the wavefunction correspond to half the expectation values, due to our coherent state labeling. Thus, we find that $x \star$ and $p \star$ generate translations of the expectation values, which is certainly the right feature to have. To better appreciate these results, one can also think about a sort of "Heisenberg picture" for the symmetry transformations as translations of the observables instead of states giving the same transformations of the expectation values. One can see that the differential operators play an important role as operators on $\tilde{\mathcal{K}}$. We can consider the set of $x_{i}, p_{i} \quad \tilde{x}_{i}$ and $\tilde{p}_{i}$ as the fundamental set of operators-functions of which essentially describe the full algebra of observables-versus the case on $\mathcal{K}$ for which the set is given only by $x_{i} \star$ and $p_{i} \star$. Note that the only nonzero commutators among the set are given by

$$
\left[x_{i}, \tilde{p}_{j}\right]=\left[p_{i}, \tilde{x}_{j}\right]=-2 i \delta_{i j} .
$$

The similar fundamental set of operators was long ago introduced within the Koopman-von Neumann formulation [48]. We see here the analogous structure in the quantum setting. For a generic $\alpha\left(p^{i}, x^{i}\right)$, the function itself (i.e. the simple multiplicative action $\left.M_{\alpha}\right), \hat{\alpha}^{L}, \hat{\alpha}^{R}$, and $\tilde{\alpha}$ are all operators to be considered on $\tilde{\mathcal{K}}$, though only two among the four are linearly independent.

Consider $G_{\omega^{i j}}=\left(x_{i} p_{j}-x_{j} p_{i}\right)$. We have

$$
\begin{gathered}
G_{\omega^{i j}} \star= \\
\left(x_{i} p_{j}-i x_{i} \partial_{x^{j}}+i p_{j} \partial_{p^{i}}+\partial_{x^{j}} \partial_{p^{i}}\right)-(i \leftrightarrow j), \\
\tilde{G}_{\omega^{i j}}=-2 i\left(x_{i} \partial_{x^{j}}-p_{j} \partial_{p^{i}}\right)-(i \leftrightarrow j) .
\end{gathered}
$$

with the explicit action

$U_{\star}\left(\omega^{i j}\right) \star \phi\left(p^{\prime}, x^{\prime}\right)=\mathrm{e}^{\frac{-i \omega^{i j}}{2}\left(G_{\omega^{i j}} \star\right)} \phi\left(p^{\prime}, x^{\prime}\right)=\left\langle p^{\prime}, x^{\prime}\left|\mathrm{e}^{\frac{-i \omega^{i j}}{2} \hat{G}_{\omega^{i j}}}\right| \phi\right\rangle=\phi\left(\mathrm{e}^{\frac{i \omega^{i j}}{2} \hat{G}_{\omega^{i j}}}\left[p^{\prime}, x^{\prime}\right]\right)$, where $\hat{G}_{\omega^{i j}}=\hat{X}_{i} \hat{P}_{j}-\hat{X}_{j} \hat{P}_{i}$ is the angular momentum operator on the Hilbert space $\mathcal{H}$ and $\mathrm{e}^{\frac{i \omega^{i j}}{2} \hat{G}_{\omega^{i j}}}\left[p^{\prime}, x^{\prime}\right]$ (no sum over the $i, j$ indices) the rotated $\left(p^{\prime}, x^{\prime}\right)$. This corresponds to the coset space action [4], i.e. a rotation about the $k$-th direction of both the $p$ and $x$ as three dimensional vectors ${ }^{8}$. Together with ${ }^{8}$ Note that $\hat{G}_{\omega^{j}}$ carries the units of $\hbar$, which are taken as 2 . Hence, for the dimensionless parameter $\omega^{i j}, \frac{i \omega^{i j}}{2} \hat{G}_{\omega^{j}}$ with 2 standing in for $\hbar$, is the right dimensionless rotation operator. A rotation on the $p$ or $x$ vector corresponds to the same rotation on $2 p$ or $2 x$ as the expectation values. 
the $G_{x^{i}}$ and $G_{-p^{i}}$ (and $G_{\theta}$ ) parts, we have the full set of operators from the generators of the $H_{R}(3)$ subgroup of the $U(1)$ extended Galilean symmetry with the time translation taken out. This portion of the ten generators $G_{s}$, is of course a commutative set. The set of $G_{s}^{L}=G_{s} \star$ represents the symmetry on $\mathcal{K}$, and constitute a subalgebra of the algebra of physical observables. We can easily see that the $G_{s}^{R}$ set does the same as a right action. A $G_{s}^{L}$ always commutes with a $G_{s^{\prime}}^{R}$ since, in general, $\left[\hat{\alpha}^{L}, \hat{\gamma}^{R}\right]=0$. Explicitly, we have

$$
\begin{gathered}
{\left[G_{\omega^{i j}}^{L / R}, G_{\omega^{h k}}^{L / R}\right]= \pm 2 i\left(\delta_{j k} G_{\omega^{i h}}^{L / R}-\delta_{j h} G_{\omega^{i k}}^{L / R}+\delta_{i h} G_{\omega^{j k}}^{L / R}-\delta_{i k} G_{\omega^{j h}}^{L / R}\right),} \\
{\left[G_{\omega^{j i}}^{L / R}, G_{-x^{k}}^{L / R}\right]= \pm 2 i\left(\delta_{j k} G_{-x^{i}}^{L / R}-\delta_{i k} G_{-x^{j}}^{L / R}\right),} \\
{\left[G_{\omega^{j i}}^{L / R}, G_{p^{k}}^{L / R}\right]= \pm 2 i\left(\delta_{j k} G_{p^{i}}^{L / R}-\delta_{i k} G_{p^{j}}^{L / R}\right),} \\
{\left[G_{p^{i}}^{L / R}, G_{-x^{j}}^{L / R}\right]= \pm 2 i \delta_{i j} G_{\theta}^{L / R}} \\
{\left[G_{p^{i}}^{L / R}, G_{p^{j}}^{L / R}\right]=\left[G_{-x^{i}}^{L / R}, G_{-x^{j}}^{L / R}\right]=0,}
\end{gathered}
$$

and $G_{\theta}^{L / R}=1$ commutes with all other generators. Note that the factors of 2 are really taking the place of $\hbar$ because of the choice of units. The upper and lower signs correspond to the $G_{s}^{L}$ and $G_{s}^{R}$ results, respectively. For the $\tilde{G}_{s}$ set, we can see that the set of commutators is same as that of $G_{s}^{L}$ with however the vanishing $\tilde{G}_{\theta}$ giving a vanishing $\left[\tilde{G}_{p^{i}}, \tilde{G}_{-x^{j}}\right]$. As a result, we can also see the $\tilde{G}_{s}$ set without $\tilde{G}_{\theta}$ as giving the symmetry without the central extension, similar to the classical case. Besides, we have

$$
\begin{gathered}
{\left[G_{\omega^{i j}}, \tilde{G}_{\omega^{h k}}\right]=2 i\left(\delta_{j k} G_{\omega^{i h}}-\delta_{j h} G_{\omega^{i k}}+\delta_{i h} G_{\omega^{j k}}-\delta_{i k} G_{\omega^{j h}}\right),} \\
{\left[G_{\omega^{i j}}, \tilde{G}_{-x^{k}}\right]=-2 i\left(\delta_{j k} G_{-x^{i}}-\delta_{i k} G_{-x^{j}}\right),} \\
{\left[G_{\omega^{i j}}, \tilde{G}_{p^{k}}\right]=-2 i\left(\delta_{j k} G_{p^{i}}-\delta_{i k} G_{p^{j}}\right),} \\
{\left[\tilde{G}_{\omega^{j j}}, G_{-x^{k}}\right]=-2 i\left(\delta_{j k} G_{-x^{i}}-\delta_{i k} G_{-x^{j}}\right),} \\
{\left[\tilde{G}_{\omega^{i j}}, G_{p^{k}}\right]=-2 i\left(\delta_{j k} G_{p^{i}}-\delta_{i k} G_{p^{j}}\right),} \\
{\left[G_{p^{i}}, \tilde{G}_{-x^{j}}\right]=-\left[G_{-x^{i}}, \tilde{G}_{p^{j}}\right]=2 i \delta_{i j},} \\
{\left[G_{p^{i}}, \tilde{G}_{p^{j}}\right]=\left[G_{-x^{i}}, \tilde{G}_{-x^{j}}\right]=0 .}
\end{gathered}
$$

The time translation generator $G_{t}^{L / R}$, needed to complete the above set of ten $G_{s}^{L R}$ into the full extended Galilean symmetry, is given by $G_{t}=\frac{p^{i} p_{i}}{2 m}$ where $m$ is the particle mass. One can see that $G_{t}^{L / R}$ commutes with each generator, except for having ${ }^{9}$

${ }^{9}$ The usual presentation of the symmetry uses the Galilean boost generators $K_{i}$ in place of $X_{i}$, which corresponds to $G_{p^{i}}^{L R}$ here. We have $K_{i}$ be matched to $m G_{p^{i}}^{L R}$. 


$$
\left[G_{p^{i}}^{L / R}, G_{t}^{L / R}\right]= \pm \frac{2 i}{m} G_{-x^{i}}^{L / R} .
$$

Similarly, we have $\tilde{G}_{t}=\frac{-2 i}{m} p^{i} \partial_{x^{i}}$ giving $\left[\tilde{G}_{p^{i}}, \tilde{G}_{t}\right]=\frac{2 i}{m} \tilde{G}_{-x^{i}}$. A generic Hamiltonian for a particle would have $G_{t}=\kappa$ to be given with an extra additive part as the potential energy $v(p, x)=v(x)$. It is also of some interest to illustrate explicitly the Heisenberg equation of motion in considerations of evolution, both in $\mathcal{K}$ and $\tilde{\mathcal{K}}$. For the time dependent operator $\alpha(p, x ; t) \star$, on $\mathcal{K}$ we have $\frac{\mathrm{d}}{\mathrm{d} t} \alpha \star=\frac{1}{2 i}[\alpha \star, \kappa \star]$, while for $\alpha(p, x ; t)$ on $\tilde{\mathcal{K}}$ we have

$$
\frac{\mathrm{d}}{\mathrm{d} t} \alpha \star=\frac{1}{2 i}[\alpha \star, \tilde{\kappa}]=\frac{1}{2 i}\left(\hat{\alpha}^{L} \hat{\kappa}^{L}-\hat{\alpha}^{L} \hat{\kappa}^{R}-\hat{\kappa}^{L} \hat{\alpha}^{L}+\hat{\kappa}^{R} \hat{\alpha}^{L}\right)=\frac{1}{2 i}[\alpha \star, \kappa \star],
$$

where $\tilde{\kappa} \equiv \hat{\kappa}^{L}-\hat{\kappa}^{R}=\tilde{G}_{t}$; hence, we arrive at the same equation as that on $\mathcal{K}$. This equation can simply be written as

$$
\frac{\mathrm{d}}{\mathrm{d} t} \alpha=\frac{1}{2 i}\{\alpha, \kappa\}_{\star} .
$$

Taking $\tilde{\kappa}=\frac{-2 i}{m} p^{i} \partial_{x^{i}}+\tilde{v}$ explicitly and applying this to the observables $x^{i}$ and $p^{i}$, we have

$$
\frac{\mathrm{d}}{\mathrm{d} t} \alpha=\frac{p^{i}}{m} \partial_{x^{i}} \alpha-\sum_{n \text { odd }} \frac{i^{n-1}}{n !}\left(\partial_{p_{i}}^{n} \alpha\right) \partial_{x^{i}}^{n} v .
$$

$v$ with vanishing third derivatives, or $\alpha$ with $\partial_{p_{i}}^{n} \alpha=0$, provide particularly important examples of the equation $\alpha$ being $x^{i}$ and $p^{i}$. The equation reduces to a form exactly the same as the one for $\alpha$, and $\kappa$, as if it is a classical observable.

\section{To The Relativity Symmetry at the Classical Limit}

We have presented the formulation of the classical limit of quantum mechanics from the perspective of a contraction of the relativity symmetry, and the corresponding representations, in Ref. [4] within the Hilbert space picture on $\mathcal{H}$ and $\mathcal{K}$. In Section 2, we have presented a formulation within the WWGM setting, focusing on the key part of the Heisenberg-Weyl subgroup. We are now going to push that to the full relativity symmetry. Taking the full extended Galilean symmetry with abstract generators $X_{i}, P_{i}, J_{i j}, H$ and $I$ as represented on $\mathcal{K}$ by the set of eleven $G_{s} \star\left(=G_{s}^{L}\right)$ above, the contraction is to be given by $X_{i}^{c}=\frac{\sqrt{\hbar}}{k} X_{i}, P_{i}^{c}=\frac{\sqrt{\hbar}}{k} P_{i}, J_{i j}^{c}=\frac{\hbar}{2} J_{i j}, H^{c}=\frac{\hbar}{2} H$, and $I^{c}=I$ taken to the $k \rightarrow \infty$ limit. Note that setting $k=1$ gives the usual commutator set with an explicit $\hbar$ (in the place of the factor 2), which can be considered as having the generators in the usual, classical system of units. Again, we take the contraction of the representation(s), with $x^{c}$ and $p^{c}$ standing in for $x$ and $p$. As the whole algebra of $\alpha(p, x) \star$ reduces to the Poisson algebra $\alpha\left(p^{c}, x^{c}\right)$ of clas- 
sical observables $\alpha\left(p^{c}, x^{c}\right)$, all of the $G_{s}(p, x) \star$ yield the $G_{s}\left(p^{c}, x^{c}\right)$, which all commute among themselves. The noncommutative observable algebra for the Hilbert space of pure states $\mathcal{K}$, upon the symmetry contraction, reduces to a commutative algebra as a result of the reduction of $\mathcal{K}$ to the simple sum of one-dimensional subspaces of each coherent state [4]. Each $\alpha\left(p^{c}, x^{c}\right)$ is diagonal on the resulting Hilbert space of pure states, which contains only the delta functions. How do we recover the noncommutative relativity symmetry at the classical level then, either on the observable algebra or in the Koopman-von Neumann formulation? The answer is to be found from the Tomita representation picture of the Hilbert space $\tilde{\mathcal{K}}$. The Koopman Hilbert space essentially comes from $\tilde{\mathcal{K}}$.

Take the set of $\tilde{G}_{s}$, we have

$$
\begin{gathered}
\tilde{G}_{\omega^{i j}}^{c}=\frac{\hbar}{2} \tilde{G}_{\omega^{i j}}=-i \hbar\left(x_{i}^{c} \partial_{x^{j c}}-p_{j}^{c} \partial_{p^{i c}}-x_{j}^{c} \partial_{x^{i c}}+p_{i}^{c} \partial_{p^{j c}}\right), \\
\tilde{G}_{t}^{c}=\frac{\hbar}{2} \tilde{G}_{t}=\frac{-i \hbar}{m} p_{i} \partial_{x_{i}}=\frac{-i \hbar}{m} p_{i}^{c} \partial_{x_{i}^{c}},
\end{gathered}
$$

and again $\tilde{G}_{\theta}^{c}=\tilde{G}_{\theta}=0$. These results are independent of the contraction parameter $k$, in fact, they are independent of $\frac{p}{p^{c}}=\frac{x}{x^{c}}$. The unitary operators can be written as

$$
\tilde{U}_{\star}(\omega)=\mathrm{e}^{\frac{-i}{\hbar} \omega^{i j} \tilde{G}_{\omega}^{c}}, \quad \tilde{U}_{\star}(t)=\mathrm{e}^{\frac{-i}{\hbar} \tilde{G}_{t}^{c}} .
$$

Similarly, if we take $\tilde{G}_{p}^{c}=\frac{\sqrt{\hbar}}{k} \tilde{G}_{p}=\frac{2 i \hbar}{k^{2}} \partial_{p^{c}}$ and $\tilde{G}_{-x}^{c}=\frac{\sqrt{\hbar}}{k} \tilde{G}_{-x}=\frac{2 i \hbar}{k^{2}} \partial_{x^{c}}$ (we drop the spatial index in $x$ and $p$ for simplicity, similarly for $\omega$ above), the results vanish in the $k \rightarrow \infty$ limit. This seems to create a problem, however, we are not interested in the operators generating translations in $p$ and $x$. We should be looking at translations in $p^{c}$ and $x^{c}$, i.e. rewriting $\tilde{U}_{\star}(p)$ and $\tilde{U}_{\star}(x)$ as $\tilde{U}_{\star}\left(p^{c}\right)$ and $\tilde{U}_{\star}\left(x^{c}\right)$. Introducing generators $\tilde{G}_{p^{c}}^{c}$ and $\tilde{G}_{-x^{c}}^{c}$ satisfying

$$
\mathrm{e}^{\frac{-i}{\hbar} p^{c} \tilde{G}_{p}^{c}}=\tilde{U}_{\star}\left(p^{c}\right)=\mathrm{e}^{\frac{-i p}{2} \tilde{G}_{p}}, \quad \mathrm{e}^{\frac{-i}{\hbar}\left(-x^{c}\right) \tilde{G}_{-x}^{c}}=\tilde{U}_{\star}\left(x^{c}\right)=\mathrm{e}^{\frac{i x}{2} \tilde{G}_{-x}},
$$

we can see that

$$
\tilde{G}_{p^{c}}=i \hbar \partial_{p^{c}}, \quad \tilde{G}_{-x^{c}}=i \hbar \partial_{x^{c}},
$$

which are again independent of $\frac{p}{p^{c}}=\frac{x}{x^{c}}$, and therefore independent of $k$. Note that $\tilde{G}_{p^{c}}$ and $\tilde{G}_{x^{c}}$ are exactly the invariant vector fields of the manifold of $\left(p^{c}, x^{c}, \theta\right)$ corresponding to the contracted symmetry from the HeisenbergWeyl group. To summarize, we have the set of $\tilde{G}_{\omega}^{c}, \tilde{G}_{t}^{c}, \tilde{G}_{p^{c}}^{c}, \tilde{G}_{-x^{c}}^{c}$, and $\tilde{G}_{\theta}^{c}$ giving the commutators exactly as the as the old set of $\tilde{G}_{\omega}, \tilde{G}_{t}, \tilde{G}_{p}, \tilde{G}_{-x}$, and $\tilde{G}_{\theta}$, with the factors of 2 all replaced by $\hbar$. With $\tilde{G}_{\theta}$ taken out, the rest constitute a representation of the contracted Galilean symmetry without the $U(1)$ 
central extension, which at the abstract Lie algebra level is trivialized and decoupled from the rest.

Next, take the multiplicative operators $G_{\omega^{i j}}^{c}=x_{i}^{c} p_{j}^{c}-x_{j}^{c} p_{i}^{c}, G_{t}^{c}=\frac{p_{i}^{c} p^{i c}}{m}$, $G_{p^{c}}^{c}=x^{c}$, and $G_{-x^{c}}^{c}=p^{c}$. We have the formal relation

$$
\begin{aligned}
& G_{\omega^{i j}}^{c}=\frac{\hbar}{k^{2}} G_{\omega^{i j}}, \quad G_{t}^{c}=\frac{\hbar}{k^{2}} G_{t}, \\
& G_{p^{c}}^{c}=\frac{\sqrt{\hbar}}{k} G_{p}, \quad G_{-x^{c}}^{c}=\frac{\sqrt{\hbar}}{k} G_{-x} .
\end{aligned}
$$

The commutator results for the classical operators with the $\tilde{G}_{s^{c}}^{c}$ (with $\omega^{c}=\omega$ and $t^{c}=t$ ) set above correspond again to results in Equation (75) with 2 replaced $\hbar$. Thus, we recover the full algebraic structure introduced in Ref. [48] for the Koopman-von Neumann classical setting.

\section{To the Koopman-Von Neumann Classical Dynamics}

Finally, we check the explicit dynamical description obtained for the classic setting, focusing especially on the Koopman-von Neumann formulation. The Schrödinger equation, Heisenberg equation, and Liouville equation are to be cast in the following forms in the contraction limit

$$
\begin{gathered}
i \hbar \frac{\mathrm{d}}{\mathrm{d} t} \phi\left(p^{c}, x^{c} ; t\right)=\frac{k^{2}}{2} \kappa^{c}\left(p^{c}, x^{c}\right) \star^{c} \phi\left(p^{c}, x^{c} ; t\right) \rightarrow \infty, \\
\frac{\mathrm{d}}{\mathrm{d} t} \alpha\left(p^{c}, x^{c} ; t\right)=\frac{k^{2}}{2 i \hbar}\left\{\alpha\left(p^{c}, x^{c} ; t\right), \kappa^{c}\left(p^{c}, x^{c}\right)\right\}_{\star^{c}} \rightarrow\left\{\alpha\left(p^{c}, x^{c} ; t\right), \kappa^{c}\left(p^{c}, x^{c}\right)\right\}, \\
\frac{\mathrm{d}}{\mathrm{d} t} \rho\left(p^{c}, x^{c} ; t\right)=\frac{k^{2}}{2 i \hbar}\left\{\kappa^{c}\left(p^{c}, x^{c}\right), \rho\left(p^{c}, x^{c} ; t\right)\right\}_{\star^{c}} \rightarrow\left\{\kappa^{c}\left(p^{c}, x^{c}\right), \rho\left(p^{c}, x^{c} ; t\right)\right\},
\end{gathered}
$$

with the classical (antisymmetric) Poisson bracket $\{\cdot, \cdot\}$ of classical phase space coordinates $\left(p^{c}, x^{c}\right)$. So, the Schrödinger equation on the Hilbert space of pure state fails to make sense at the contraction limit, while the Heisenberg equation and the Liouville equation give the correct classical results. The problem of the Schrödinger equation is not beyond expectations. The Hilbert space of pure states, as an irreducible unitary representation, collapses to the simple sum of one-dimensional subspaces of the coherent states, so there is no continuous evolution to described on it any more. Recall that the Heisenberg equation can be seen as one on $\tilde{\mathcal{K}}$, and hence it survives. Moreover, the Liouville equation is the Schrödinger equation on $\tilde{\mathcal{K}}$. The reducible representation is on the Hilbert space $\tilde{\mathcal{K}}$ containing all the states-pure or mixed-and therefore it is not at all bothered by the fact that most of the pure quantum states become mixed states in the classical limit. Furthermore, note that the (classical) Liouville equation is insensitive to the rescaling/renormalization of $\rho$ to $\rho^{c}$, and similarly for the classical equation of motion going from $\alpha$ to $\alpha^{c}$.

In the Koopman-von Neumann formulation, a classical wavefunction $\phi^{c}$ is to be introduced with $\rho^{c} \equiv\left|\phi^{c}\right|^{2}$ for each $\rho^{c}$. Each $\phi^{c}$ describes a mixed 
state in general, as does $\rho^{c}$. The Koopman-von Neumann Hilbert space is one of a reducible representation, A Koopman-Schrödinger equation for $\phi^{c}$ relating to the classical Liouville equation $\frac{\mathrm{d}}{\mathrm{d} t} \rho^{c}=\left\{\kappa^{c}, \rho^{c}\right\}$ can be written then as

$$
i \hbar \frac{\mathrm{d}}{\mathrm{d} t} \phi^{c}=\kappa^{c} \phi^{c} .
$$

One can rewrite the classical equation of motion in the Koopman-Heisenberg form [36] as

$$
\frac{\partial}{\partial t} M_{\alpha}=M_{\left\{\kappa^{c}, \alpha\right\}}=\left[X_{\kappa^{c}}, M_{\alpha}\right] \text {, }
$$

where $X_{\kappa}=\left[\frac{\partial \kappa}{\partial p_{i}^{c}} \frac{\partial}{\partial x^{i c}}-\frac{\partial \kappa}{\partial x^{i c}} \frac{\partial}{\partial p_{i}^{c}}\right]$ is the (classical) Hamiltonian vector field, which gives

$$
M_{\alpha(t)}=\mathrm{e}^{i t\left(-i X_{\kappa^{c}}\right)} M_{\alpha} \mathrm{e}^{-i t\left(-i X_{\kappa^{c}}\right)}=\mathrm{e}^{t X_{\kappa^{c}}} M_{\alpha} \mathrm{e}^{-t X_{\kappa^{c}}} .
$$

Recall that the multiplicative operator $M_{\alpha}=\alpha$ is just the classical limit of the $\alpha \star$ [cf. Equation (30)]; hence it is a simple multiplication with $\alpha\left(p^{c}, x^{c}\right)$ on the classical Hilbert space $L^{2}(\Pi)$. Taking a closer look, we see that the solution to the equation of motion (84) before taking $k \rightarrow \infty$ can be written as

$$
\alpha(t) \star^{c}=\mathrm{e}^{\frac{k^{2}}{2 \hbar} i\left(\kappa^{c} \star^{c}\right)}\left[\alpha(0) \star^{c}\right] \mathrm{e}^{-\frac{k^{2}}{2 \hbar} i t\left(\kappa^{c} \star^{c}\right)} .
$$

This equation is nothing other than the $\alpha(t) \star$ solution to the original Heisenberg equation of (78) written in terms of the rescaled classical variables. Expanding Equation (53) and keeping only the first two terms, we have

$$
U_{\star}(t)=\mathrm{e}^{-\frac{k^{2}}{2 \hbar} i t\left(\kappa^{c} \star^{c}\right)} \rightarrow \mathrm{e}^{-\frac{k^{2}}{2 \hbar} i t\left(\kappa^{c}-\frac{2 i \hbar}{k^{2}} X_{\kappa}^{c}\right)}=\mathrm{e}^{-\frac{i k^{2} t}{2 \hbar} \kappa^{c}} \mathrm{e}^{-t X_{\kappa}^{c}} .
$$

This result is obviously consistent with Equation (88), as the first exponential factor simply cancels itself out. The classical limit is taken as the $k \rightarrow \infty$ limit, but the dynamics is determined by the noncommutative part of the star product; therefore it is determined by the first nontrivial term in the expansion, which is also the dominating real term. For the Schrödinger picture considerations, however, one would keep only the dominating first term. The limit $U_{\star}(t)$ is then consistent with the limiting Schrödinger equation, but both involve the diverging $k^{2}$ factor. Again, the quantum Schrödinger equation is an equation of motion for the pure states the classical limit, and there do not form a connected set in the reduced Hilbert space (except formally at the zero magnitude point). The Koopman-Schrödinger equation is exactly given by putting $k^{2}=2$ back into the limiting Schrödinger equation for the diverging $k$.

The solution to the Koopman-Schrödinger equation is given in terms of the Koopman-Schrödinger flow $U^{K S}(t)=\mathrm{e}^{\frac{-i t}{\hbar} \mathcal{G}_{\kappa}}$ in Ref. [36] with the generator $\mathcal{G}_{\kappa}$ given by 


$$
\mathcal{G}_{\kappa}=M_{\kappa}+M_{\vartheta(\kappa)}-i \hbar X_{\kappa} .
$$

The first two terms contribute a change of a complex phase for $\phi^{c}$ with no effect in the Heisenberg picture. The last term, and thus the whole set of $U^{K S}(t)$, gives the Koopman-Heisenberg equation we obtained above, as well as a time translation of (the magnitude of) $\phi^{c}$ in the Schrödinger picture. The $M_{\vartheta(\kappa)}$ part is responsible for the geometric phase [49] [50], a notion which requires formulating states, quantum or classical, as sections of a $U(1)$ principal bundle or a Hermitian line bundle [49] [50] [51] for its description. $M_{\vartheta(\alpha)}-i \hbar X_{\alpha}$ is really a covariant derivative ( $\vartheta$ a connection form) associated to the function $\alpha\left(p^{c}, x^{c}\right)$ which guarantees $\mathcal{G}_{\{\alpha, \beta\}}=i \hbar\left[\mathcal{G}_{\alpha}, \mathcal{G}_{\beta}\right]$, i.e. the operators $\mathcal{G}_{\alpha}$ form a representation of same Lie algebra as the Poisson algebra. Adopting the canonical trivialization of the $U(1)$ bundle over $\Pi$, coordinated by $p_{i}^{c}-d x^{i c}$ as a Kähler manifold with a Euclidean metric on $\left(d \vartheta=d x^{i c} \wedge d p_{i}^{c}\right.$ is the symplectic form $)^{10}, \quad \mathcal{G}_{\alpha}$ can be taken as acting on the wavefunction $\phi^{c} \in L^{2}(\Pi)$ with $\vartheta(\alpha)=-\frac{1}{2}\left[p_{i}^{c} \frac{\partial \kappa}{\partial p_{i}^{c}}+x_{i}^{c} \frac{\partial \kappa}{\partial x_{i}^{c}}\right]$ [52]. It would be interesting to see a full $U(1)$ bundle formulation of the WWGM formalism and its contraction limit, which is however beyond the scope of this article.

\section{Conclusions}

We have explicitly presented a version of the WWGM formalism for quantum mechanics, which we propose as the most natural prescription unifying, the formalism most familiar to a general physicist (the one base on a Hilbert space of wavefunctions) and the abstract mathematical algebraic formalism related to noncommutative geometry. On the (pure state) Hilbert space $\mathcal{K}$ of wavefunctions $\phi\left(p_{i}, x_{i}\right)$ from the canonical coherent state basis, the observable algebra as a functional algebra of the $P_{i}$ and $X_{i}$ operators $C\left(P_{i}, X_{i}\right)$ can be seen as both the operator (functional) algebra $C\left(p_{i} \star, x_{i} \star\right)$ as well as $C\left(p_{i}, x_{i}\right)$ with a Moyal star product; $\alpha\left(p_{i} \star, x_{i} \star\right) \phi=\alpha\left(p_{i}, x_{i}\right) \star \phi$. We advocate the former picture and the important notion that the algebra is essentially an irreducible (cyclic) representation of the group $(C-)$ algebra from the relativity symmetry within which the Hilbert space is a representation for the group. The modern mathematics of noncommutative geometry [1] [53] [54] essentially says that the noncommutative algebra $C\left(p_{i} \star, x_{i} \star\right)$ is to be seen as an algebra of continuous functions of a geometric/topological space with the six noncommutative coordinates $p_{i} \star$ and $x_{i} \star$, and coordinates are of course the basic observables in terms of which all other observables can be constructed. $C\left(p_{i} \star, x_{i} \star\right)$ as a $C$-algebra corresponds to the set of compact operators on $\mathcal{K}$ is a Moyal subalgebra of $\mathbb{B}(\mathcal{K})$ as given by $\mathcal{M}^{\prime}$ (which is a $W^{\prime}$-algebra). The mathematics also offers another geometric object as a kind of dual object to the $C$-algebra, ${ }^{10}$ The metric is essentially the restriction of the Fubini-Study metric on the quantum phase space (the projective Hilbert space) as the Kähler manifold $C P^{\infty}$ to the coherent state submanifold [33]. It is hence totally compatible with the quantum description. 
namely the space of pure states $\omega_{\phi}[22]$, which is equivalent to the (projective) Hilbert space (of $\mathcal{K}$ ) [55]. The projective Hilbert space is the infinite-dimensional Kähler manifold $C P^{\infty}$ [33] [56], with a set of "six times $\infty$ " homogeneous coordinates. One key purpose of the article is to help a general physicist to appreciate such a perspective. Of course such an algebraic-geometric perspective also works perfectly well with Newtonian physics for which the observable algebra is commutative and contains functions of the classical phase space coordinates. We illustrate here also how that classical limit is retrieved from the quantum one.

This geometric notion is usually considered as only about the quantum phase space. Actually, the standard description of quantum mechanics breaks the conceptual connection between the phase space, the configuration space, and physical space itself in classical mechanics-physical space is the configuration space (all possible positions) of a free particle, or of the center of mass as a degree of freedom for a closed system of particles; the configuration space is sort of like half the phase space, with the other half being the momentum space of conjugate variables. However, from both the noncommutative geometry picture and the $C P^{\infty}$ picture (for a single quantum particle) discussed above, it certainly looks like it does not have to be the case. In particular, $C P^{\infty}$ is a symplectic manifold and the Schrödinger equation is an infinite set of Hamiltonian equations of motion with the configuration and conjugate momentum variables taken as, say, the real and imaginary parts of $\phi\left(p_{i}, x_{i}\right)$ at each $\left(p_{i}, x_{i}\right)$ (or those of $\left\langle\phi \mid a_{n}\right\rangle$ for any set $\left|a_{n}\right\rangle$ of orthonormal basis). In Ref. [4], we have constructed a quantum model of physical space, or the position/configuration space of a particle, along parallel lines of the coherent state phase space construction as a representation of the relativity symmetry. Moreover, we showed that the model reduces back to the Newtonian model as the classical limit formulated as a relativity symmetry contraction limit. Part of the analysis in the current article was motivated by the idea of illustrating the solid dynamical picture underlying that framework.

The quantum physical space obtained in Ref. [4] is actually a Hilbert space equivalent to that of the phase space. The key reason is that for the quantum relativity symmetry $\tilde{G}(3)$ as the $U(1)$ central extension of the classical Galilean symmetry $G(3)$, phase space representations are generally irreducible while in the classical case they may be reduced to a sum of the position/configuration space and the momentum space ones. The central charge generator, as the $X_{i}-P_{i}$ commutator generates, a complex phase rotation in relation to the natural complex structure in $X_{i}+i P_{i}$ with the complex coordinates $\phi\left(p_{i}, x_{i}\right)$ mixing the position/configuration coordinates with the momentum coordinates, the division of which would otherwise be respected by the other relativity symmetry transformations. The analysis here establishes explicitly that the $\tilde{G}(3)$ group plays the full role of a relativity symmetry for quantum mechanics with the quantum model of the physical space and gives all the corresponding aspects for the Newtonian theory as an approximation to be described as a relativity 
symmetry contraction. The results also gives a comprehensive treatment of the classical limit of quantum mechanics, to which there are otherwise quite some confusing notions about in the literature.

The explicit analysis in Ref. [4] focused only on the $H_{R}(3)$ subgroup with the time translation generator taken out, which is good enough for the mostly kinematical considerations there. Along these lines, we put strong emphasis on the (relativity) symmetry group as the starting point. The observable algebra is essentially the group $\left(C^{*}\right)$ algebra or an irreducible representation of it. Actually, we focus only on the Heisenberg-Weyl subgroup $H(3)$, and take into consideration the full relativity symmetry $\tilde{G}(3)$ only as unitary transformation on the Hilbert space and as automorphisms on the observable algebra. All of this works very well because the relevant (e.g. spin zero) representation of the $\tilde{G}(3)$ group algebra is contained in $C\left(p_{i} \star, x_{i} \star\right)$. This is a natural parallel to the Hilbert space of pure states as an representation of $H(3)$ and $H_{R}(3)$ (or $\tilde{G}(3)$ ). This is more or less the physical statement that (orbital) angular momentum and Hamiltonian variables/operators are to be written in terms of the position and momentum ones. It is really a consequence of the structure of $\tilde{G}(3)$ with the series of invariant subgroups

$$
U(1) \prec H(3) \prec H_{R}(3) \prec \tilde{G}(3),
$$

giving the following semidirect product structures:

$$
\tilde{G}(3)=H(3) \rtimes(S O(3) \times T),
$$

where $T$ denotes the one parameter group of time translations. The other relativity transformations act on $H(3)$ as outer automorphisms and on its group algebra as inner automorphisms. Again, the Hilbert space as a group representation naturally sits inside the representation of the group algebra with the natural (noncommutative, algebraic) multiplicative actions of the latter as the operator actions.

It is also interesting that while the rotational symmetry $S O(3)$ is naturally to be included in the mathematical picture of even just the $H(3)$ symmetry, the Galilean time translation is not. Moreover, we have no problem describing the transformations generated by any real/Hermitian Hamiltonian function $\kappa\left(p_{i}, x_{i}\right)$ or operator $\kappa\left(p_{i}, x_{i}\right) \star$ as unitary transformations on the Hilbert space and automorphisms of the observable algebra, just like any Hamiltonian flow on a symplectic manifold. But then there is no reason to single out the parameter of a particular Hamiltonian flow as physical time and the generator of physical energy. We may have to look for a more natural relativity symmetry framework in order to truly understood time, for example with Lorentz symmetry incorporated. For the current authors, we are particularly interested in using the relativity symmetry as the basic key mathematical structure, and plan on pushing forward for models of quantum spacetime and its related dynamics on the deep microscopic scale based on the idea of relativity symmetry deformation/stabilization [17]. 
In summary, quantum mechanics can be, and we believe should be, seen as a theory of particle dynamics on a quantum/noncommutative model of the physical space with a picture as the infinite dimensional Kähler manifold $C P^{\infty}$. It has a relativity symmetry of $\tilde{G}(3)$ and the observable algebra is naturally the representation of the group $C$-algebra corresponding to the representation of $\tilde{G}(3)$ (time-independent spin-zero) that describes the physical space. The WWGM formalism is just such a representation theory, and hence also essentially the Hilbert space theory. Dynamics is included in the Hamiltonian flows on $C P^{\infty}$ as well as the corresponding automorphism flows on the $C$-algebra. The mathematical framework is valid for any group as relativity symmetry, and a group obtained as the contraction limit of another serves as an approximation of the latter with the full theory retrievable from pushing the contraction throughout the original theory, as our illustration of obtaining the Newtonian theory from quantum mechanics. Lie group/algebra deformations in the reverse process to contraction, hence giving natural candidates for theories the quantum and classical mechanics serves as approximation. The fully deformed/stabilized (special) relativity symmetry, probably for Planckian physics, is expected to give full noncommutativity among all $X$ and $P$ to which quantum mechanics is the minimal case with noncommutativity. It suggests all noncommutative models of spacetime should have be phase space models; energy-momentum is much a part of the physical space only in the classical approximation to which one can consider the configuration and the momentum parts separately.

\section{Acknowledgements}

The authors are partially supported by research grant MOST 105-2112-M-008017 and MOST 106-2112-M-008-008 from the MOST of Taiwan.

\section{Conflicts of Interest}

The authors declare no conflicts of interest regarding the publication of this paper.

\section{References}

[1] Connes, A. (1994) Noncommutative Geometry. Academic Press, Cambridge.

[2] Doplicher, S., Fredenhagen, K. and Roberts, J.E. (1995) The Quantum Structure of Space-Time at the Planck Scale and Quantum Fields. Communications in Mathematical Physics, 172, 187-220. https://doi.org/10.1007/BF02104515

[3] Seiberg, N. and Witten, E. (1999) String Theory and Noncommutative Geometry. Journal of High Energy Physics, 9, 32.

https://doi.org/10.1088/1126-6708/1999/09/032

[4] Chew, C.-S., Kong, O.C.W. and Payne, J. (2017) A Quantum Space Behind Simple Quantum Mechanics. Advances in High Energy Physics, 2017, Article ID: 4395918. https://doi.org/10.1155/2017/4395918

[5] Snyder, H.S. (1947) Quantized Space-Time. Physical Review, 71, 38-41. https://doi.org/10.1103/PhysRev.71.38 
[6] Yang, C.N. (1974) On Quantized Space-Time. Physical Review, 72, 874. https://doi.org/10.1103/PhysRev.72.874

[7] Mendes, R.V. (1994) Deformations, Stable Theories and Fundamental Constants. Journal of Physics A, 27, 8091-8104. https://doi.org/10.1088/0305-4470/27/24/019

[8] Chryssomalakos, C. and Okon, E. (2004) Linear Form of 3-Scale Special Relativity Algebra and the Relevance of Stability. International Journal of Modern Physics D, 13, 1817-1850. https://doi.org/10.1142/S0218271804005225

[9] Chryssomalakos, C. and Okon, E. (2004) Generalized Quantum Relativistic Kinematics: A Stability Point of View. International Journal of Modern Physics D, 13, 2003-2034. https://doi.org/10.1142/S0218271804006632

[10] Amelino-Camelia, G. (2001) Testable Scenario for Relativity with Minimum Length. Physics Letters B, 510, 255-263. https://doi.org/10.1016/S0370-2693(01)00506-8

[11] Amelino-Camelia, G. (2002) Relativity in Spacetimes with Short-Distance Structure Governed by an Observer-Independent (Plankian) Length Scale. International Journal of Modern Physics D, 11, 35-59. https://doi.org/10.1142/S0218271802001330

[12] Magueijo, J. and Smolin, L. (2002) Lorentz Invariance with an Invariant Energy Scale. Physical Review Letters, 88, Article ID: 190403. https://doi.org/10.1103/PhysRevLett.88.190403

[13] Magueijo, J. and Smolin, L. (2003) Generalized Lorentz Invariance with an Invariant Energy Scale. Physical Review D, 67, Article ID: 044017. https://doi.org/10.1103/PhysRevD.67.044017

[14] Kowalski-Glikman, J. and Smolin, L. (2004) Triply Special Relativity. Physical Review D, 70, Article ID: 065020. https://doi.org/10.1103/PhysRevD.70.065020

[15] Lukierski, J. (2017) Kappa-Deformation: Historical Developments and Recent Results. Journal of Physics: Conference Series, 804, Article ID: 012028. https://doi.org/10.1088/1742-6596/804/1/012028

[16] Cho, D.N. and Kong, O.C.W. (2014) Relativity Symmetries and Lie Algebra Contractions. Annals of Physics, 351, 275-289. https://doi.org/10.1016/j.aop.2014.09.005

[17] Kong, O.C.W. (2008) A Deformed Relativity with the Quantum $\hbar$. Physics Letters $B, 665,58-61$. https://doi.org/10.1016/j.physletb.2008.05.060

[18] Kong, O.C.W. (2010) “AdS5” Geometry beyond Space-Time and 4D Noncommutative Spacetime. The QGC 2010 NIMS-APCTP 2010 International Workshop on String Theory and Cosmology, Daejeon, 30 September-2 October 2010, NCU-HEP$\mathrm{k} 040$.

[19] Kong, O.C.W. (2017) Quantum Spacetime from Quantum Mechanics to Deep Microscopic Physics. Cross-Strait Meeting on Particle Physics and Cosmology, Jinan, 20-25 April 2017, NCU-HEP-k072.

[20] Yang, H.-S. (2009) Emergent Spacetime and the Origin of Gravity. Journal of High Energy Physics, 5, 12. https://doi.org/10.1088/1126-6708/2009/05/012

[21] Aldazabal, G., Marques, D. and Nunez, C. (2013) Double Field Theory: A Pedagogical Review. Classical and Quantum Gravity, 30, Article ID: 163001. https://doi.org/10.1088/0264-9381/30/16/163001

[22] Shultz, F.W. (1982) Pure States as a Dual Object for C*-Algebras. Communications in Mathematical Physics, 82, 497-509. https://doi.org/10.1007/BF01961237

[23] Wolf, K.B. (1975) The Heiseberg-Weyl Ring in Quantum Mechanics. In: Loebl, E.M., Ed., Group Theory and Its Applications III, Academic Press, Cambridge, New York, 189-247. https://doi.org/10.1016/B978-0-12-455153-4.50010-1 
[24] Hansen, F. (1984) Quantum Mechanics in Phase Space. Reports on Mathematical Physics, 19, 361-381. https://doi.org/10.1016/0034-4877(84)90008-9

[25] Garcia-Bondia, J.M. and Varilly, J.C. (1988) Algebras of Distributions Suitable for Phase-Space Quantum Mechanics. Journal of Mathematical Physics, 29, 869-879. https://doi.org/10.1063/1.528200

[26] Dubin, D.A., Hennings, M.A. and Smith, T.B. (2000) Mathematical Aspects of Weyl Quantization and Phase. World Scientific, Singapore. https://doi.org/10.1142/4140

[27] Zachos, C.K., Fairlie, D.B. and Curtright, T.L. (2005) Quantum Mechanics in Phase Space: An Overview with Selected Papers. World Scientific, Singapore. https://doi.org/10.1142/5287

[28] Kirillov, A.A. (1972) Elements of the Theory of Representations. Springer, Berlin.

[29] Peremolov, A.M. (1977) Coherent States for Arbitrary Lie Group. Soviet Physics Uspekhi, 20, 103-117. https://doi.org/10.1070/PU1977v020n09ABEH005459

[30] Zhang, W.-M., Feng, D.H. and Gilmore, R. (1990) Coherent States: Theory and Some Applications. Reviews of Modern Physics, 62, 867-927. https://doi.org/10.1103/RevModPhys.62.867

[31] Klauder, J.R. and Sudarshan, E.C. (1968) Fundamentals of Quantum Optics. Benjamin, New York.

[32] Klauder, J.R. (2010) A Modern Approach to Functional Integration. Birkhauser, Basel. https://doi.org/10.1007/978-0-8176-4791-9

[33] Bengtsson, I. and Zyczkowski, K. (2006) Geometry of Quantum States. Cambridge University Press, Cambridge. https://doi.org/10.1017/CBO9780511535048

[34] Koopman, B.O. (1931) Hamiltonian Systems and Transformation in Hilbert Space. Proceedings of the National Academy of Sciences of the United States, 17, 315-318. https://doi.org/10.1073/pnas.17.5.315

[35] von Neumann, J. (1932) Zur Operatorenmethode in der Klassischen Mechanik. Annals of Mathematics, 33, 587-642. https://doi.org/10.2307/1968537

[36] Jauslin, H.-R. and Sugny, D. (2010) Dynamics of Mixed Classical-Quantum Systems, Geometric Quantization, and Coherent States. In: Araki, H., et al., Eds., Mathematical Horizons for Quantum Physics, World Scientific, Singapore, 65-96. https://doi.org/10.1142/9789814313322_0003

[37] Daubechies, I. and Grossmann, A. (1980) An Integral Transform Related to Quantization. Journal of Mathematical Physics, 21, 2080-2090. https://doi.org/10.1063/1.524702

[38] Stroochi, F. (2008) An Introduction to the Mathematical Structure of Quantum Mechanics. World Scientific, Singapore. https://doi.org/10.1142/7038

[39] David, F. (2015) The Formalisms of Quantum Mechanics. Springer, Berlin. https://doi.org/10.1007/978-3-319-10539-0

[40] Gilmore, R. (2005) Lie Groups, Lie Algebras, and Some of Their Applications. Dover, Mineola.

[41] Tung, W.K. (1985) Group Theory in Physics. World Scientific, Singapore. https://doi.org/10.1142/0097

[42] Takesaki, M. (1970) Tomita's Theory of Modular Hilbert Algebras and Its Applications. Springer-Verlag, Berlin. https://doi.org/10.1007/BFb0065832

[43] Grelland, H.H. (1993) Tomita Representations of Quantum and Classical Mechanics in a Bra/Ket Formulation. International Journal of Theoretical Physics, 32, 905 925. https://doi.org/10.1007/BF01215298 
[44] Grossmann, A. (1976) Parity Operator and Quantization of $\delta$-functions. Communications in Mathematical Physics, 48, 191-194. https://doi.org/10.1007/BF01617867

[45] Royer, A. (1977) Wigner Function as the Expectation Value of a Parity Operator. Physical Review A, 15, 449-450. https://doi.org/10.1103/PhysRevA.15.449

[46] Bracken, A.J., Cassinelli, G. and Wood, J.G. 2003) Quantum Symmetries and the Weyl-Wigner Product of Group Representations. Journal of Physics A: Mathematical and General, 36, 1033-1056. https://doi.org/10.1088/0305-4470/36/4/313

[47] Hakioglu, T. and Dragt, A.J. (2001) The Moyal-Lie Theory of Phase Space Quantum Mechanics. Journal of Physics A: Mathematical and General, 34, 6603-6615. https://doi.org/10.1088/0305-4470/34/34/307

[48] Loinger, A. (1962) Galilei Group and Liouville Equation, Annals of Physics, 20, 132-144. https://doi.org/10.1016/0003-4916(62)90119-7

[49] Samuel, J. and Bhandari, R. (1988) General Setting for Berry's Phase. Physical Review Letters, 60, 2339-2342. https://doi.org/10.1103/PhysRevLett.60.2339

[50] Bohm, A., Boya, L.J. and Kendrick, B. (1991) Derivation of the Geometrical Phase. Physical Review A, 43, 1206-1210. https://doi.org/10.1103/PhysRevA.43.1206

[51] Woodhouse, N. (1992) Geometric Quantization. Oxford University Press, Oxford.

[52] Tuynman, G.M. (1987) Quantization: Towards a Comparison between Methods. Journal of Mathematical Physics, 28, 2829-2840. https://doi.org/10.1063/1.527681

[53] Palmer, T.W. (2001) Banach Algebras and the General Theory of *-Algebras Vol. II. Cambridge University Press, Cambridge. https://doi.org/10.1017/CBO9780511574757

[54] Pedersen, G.K. (1979) C*-Algebras and Their Automorphism Groups. Academic Press, Cambridge.

[55] Cirelli, R., Lanzavecchia, P. and Mania, A. (1983) Normal Pure States of the von Neumann Algebra of Bounded Operators as Kahler Manifold. Journal of Physics A: Mathematical and General, 16, 3829-3835.

https://doi.org/10.1088/0305-4470/16/16/020

[56] Cirelli, R., Mania, A. and Pizzocchero, L. (1990) Quantum Mechanics as an Infinite-Dimensional Hamiltonian System with Uncertainty Structure: Part I. Journal of Mathematical Physics, 31, 2891-2897. https://doi.org/10.1063/1.528941 